\section{Nutritional Composition of Novel Plant-Based Meat Alternatives and Traditional Animal-Based Meats}

\author{
Caleb J Swing ${ }^{1}$, Tyler W Thompson', Octavio Guimaraes', Ifigenia \\ Geornaras ${ }^{1}$, Terry E Engle ${ }^{1}$, Keith E Belk ${ }^{1}$, Cody L Gifford ${ }^{2}$ and \\ Mahesh N Nair ${ }^{1 *}$
}

${ }^{1}$ Center for Meat Safety \& Quality, Department of Animal Sciences, Colorado State University, Fort Collins, USA

${ }^{2}$ Department of Animal Science, University of Wyoming, Laramie, USA

\begin{abstract}
Plant-Based Meat Alternatives (PBMA) are increasing in popularity and may provide essential nutrients for some populations. Therefore, different formulations of popular PBMAs, such as Beyond Meat Burger (BMB1 and BMB2), Impossible Foods Burger (IFB1 and IFB2), and Morning Star's Black Bean Burger (BBB), were assessed in comparison to traditional Animal-Based Meats (ABM), such as 80/20 Ground Pork (GP) for nutrient composition. Sodium content was considerably greater $(P<0.05)$ in all PBMAs than $\mathrm{GP}$, along with total saturated fat content being numerically greater when compared to GP. Vitamin E content of all PBMAs was numerically greater than ABMs. Total Essential Amino Acid (EAA) content was numerically greater in BMB2 than in ABMs, although anabolically important EAA, such as methionine and lysine were substantially greater $(P<0.05)$ in GP. While PBMAs were comparable to ABMs in many nutrients, bioavailability should be further investigated.
\end{abstract}

Keywords: Animal-based meats; Essential amino acids; Nutrition; Plant-based proteins; Saturated fatty acids

\section{Introduction}

Poor nutrition and food insecurity are widespread challenges facing the world, with global estimations suggesting that, every year, over 2 billion people may suffer from a nutrient deficiency, 690 million people may suffer from hunger, 144 million children, under the age of 5 , may suffer from stunted growth, and over 650 million people

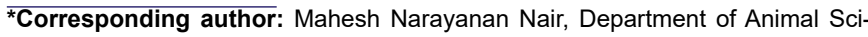
ences, Colorado State University, Fort Collins, USA, Tel: +1 9704915622; E-mail: mahesh.narayanan_nair@colostate.edu

Citation: Swing CJ, Thompson TW, Guimaraes O, Geornaras I, Engle TE, et al. (2021) Nutritional Composition of Novel Plant-Based Meat Alternatives and Traditional Animal-Based Meats. J Food Sci Nutr 7: 109.

Received: June 26, 2021; Accepted: June 30, 2021; Published: July 09, 2021

Copyright: ( $) 2021$ Swing CJ, et al. This is an open-access article distributed under the terms of the Creative Commons Attribution License, which permits unrestricted use, distribution and reproduction in any medium, provided the original author and source are credited. may suffer from obesity worldwide [1-4]. Additionally, an increasing global population, accompanied by changes in urbanization, industrialization, and globalization have compounded the challenges regarding poor nutrition and food insecurity as the global demand for animal proteins is expected to double by 2050 [5-7]. Additionally, plant-based diets, such as veganism, vegetarianism, and flexitarianism are increasing worldwide, which has generated a growing affability to plant-based diets, as well as a demand for the development of novel plant-based meat alternatives $[8,9]$.

Plant-Based Meat Alternatives (PBMA) are products that mimic the taste, appearance, or texture of Animal-Based Meats (ABM) [10]. Novel PBMAs have become popular in recent years, with the market growing at five times the rate of the ABM market in 2019 and is expected to reach a global value of $\$ 8.1$ billion by the year 2026 [11]. Commercial PBMAs are primarily being developed by companies such as Beyond Meat and Impossible Foods; however, traditionally ABM producing companies, such as Cargill, JBS, Tyson, and others are also developing novel PBMAs [12].

To mimic the elasticity and mouth feel of traditional ABMs, modern PBMAs often utilize isolated proteins obtained from legumes, oilseeds, and cereals, as well as purified fats and oils obtained from coconuts, cocoa fruit, sunflower seeds, and rapeseed $[12,13]$. While many of these plant-sourced foods can often be a good source of dietary nutrients, anti nutrient factors present in plant tissues, such as oxalates, phytates, tannins, and other antagonists, may inhibit the absorption of nutrients $[14,15]$. In contrast, ABMs have been long regarded as a good source of readily bio available nutrients; but have also been criticized as a potential risk factor for the development of various human diseases [16-18]. Therefore, a substantial knowledge gap exists about the nutrient profiles of PBMAs and ABMs, and its impact on human health and wellbeing [9].

\section{Materials and Methods}

\section{Sample collection and processing}

To adhere to the United States Department of Agriculture (USDA), Agricultural Research Service's (ARS) Food Data Central Database guidelines, original and current formulations of the Beyond Meat Burger (BMB1, BMB2), Impossible Foods Burger (IFB1, IFB2), Morning Star's Black Bean Burger (BBB), along with the $80 / 20$ Ground Pork (GP) were purchased at food service companies and supermarkets from six randomly selected cities (Seattle, WA; Peyton, CO; Memphis, TN; Newburgh, IN; Houston, TX; and Brooklyn, NY) throughout the United States with ingredients listed in table 1. Approximately $5 \mathrm{lbs}$. of frozen product, with the same lot number, were purchased and transported under refrigeration $\left(4^{\circ} \mathrm{C}\right)$ to Colorado State University (Fort Collins, CO), where they were frozen $\left(-20^{\circ} \mathrm{C}\right)$ until further analysis. Six replicates $(n=6)$ of each product (i.e., one replicated per city), designated as raw or cooked, were analyzed for nutrient content separately. Nutrient profiles for raw and cooked 80/20 Ground Beef (GB) were retrieved from the USDA-ARS Food Data Center nutrient database and utilized for comparisons in the present 
work [19,20]. Finally, Recommended Dietary Allowance (RDA) information was retrieved from the United States Department of Health and Human Services, National Institutes of Health database, and a serving size of $113 \mathrm{~g}(4 \mathrm{oz})$ was utilized for all RDA comparisons [21].

\begin{tabular}{|c|c|}
\hline Product & Ingredients \\
\hline BMB1 & $\begin{array}{l}\text { Water, Pea Protein Isolate, Expeller-Pressed Canola Oil, Refined Coconut } \\
\text { Oil, Contains } 2 \% \text { or less of the following: Cellulose from Bamboo, Meth- } \\
\text { ylcellulose, Potato Starch, Natural Flavor, Maltodextrin, Yeast Extract, } \\
\text { Salt, Sunflower Oil, Vegetable Glycerin, Dried Yeast, Gum Arabic, Citrus } \\
\text { Extract (to protect quality), Ascorbic Acid (to maintain color), Beet Juice } \\
\text { Extract (for color), Acetic Acid, Succinic Acid, Modified Food Starch, } \\
\text { Annatto (for color). }\end{array}$ \\
\hline BMB2 & $\begin{array}{l}\text { Water, Pea Protein*, Expeller-Pressed Canola Oil, Refined Coconut Oil, } \\
\text { Rice Protein, Natural Flavors, Cocoa Butter, Mung Bean Protein, Methyl- } \\
\text { cellulose, Potato Starch, Apple Extract, Pomegranate Extract, Salt, Potassi- } \\
\text { um Chloride, Vinegar, Lemon Juice Concentrate, Sunflower Lecithin, Beet } \\
\text { Juice Extract (for color). }\end{array}$ \\
\hline IFB1 & $\begin{array}{c}\text { Water, Textured Wheat Protein, Coconut Oil, Potato Protein, Natural } \\
\text { Flavors, } 2 \% \text { or less of: Leghemoglobin (Soy), Yeast Extract, Salt, Konjac } \\
\text { Gum, Xanthan Gum, Soy Protein Isolate, Vitamin E, Vitamin C, Thiamin } \\
\text { (Vitamin B1), Zinc, Niacin, Vitamin B6, Riboflavin (Vitamin B2), Vitamin } \\
\text { B12. }\end{array}$ \\
\hline IFB2 & $\begin{array}{l}\text { Water, Soy Protein Concentrate, Coconut Oil, Sunflower Oil, Natural } \\
\text { Flavors, } 2 \% \text { or less of: Potato Protein, Methylcellulose, Yeast Extract, } \\
\text { Cultured Dextrose, Food Starch Modified, Soy Leghemoglobin, Salt, Soy } \\
\text { Protein Isolate, Mixed Tocopherols (Vitamin E), Zinc Gluconate, Thiamine } \\
\text { Hydrochloride (Vitamin B1), Sodium Ascorbate (Vitamin C), Niacin, } \\
\text { Pyridoxine Hydrochloride (Vitamin B6), Riboflavin (Vitamin B2), Vitamin } \\
\text { B12. }\end{array}$ \\
\hline BBB & $\begin{array}{l}\text { Water, Onions, Cooked Black Beans (Black Beans, Water), Cooked Brown } \\
\text { Rice (Water, Brown Rice), Corn, Soy Protein Concentrate, Tomatoes, } \\
\text { Wheat Gluten, Onion Powder, Vegetable Oil (Corn, Canola, and/or Sun- } \\
\text { flower Oil), Green Chiles, Soy Protein Isolate, Bulgur Wheat, Cornstarch, } \\
\text { Contains } 2 \% \text { or less of Green Peppers, Red Bell Peppers, Spices, Tomato } \\
\text { Powder, Cilantro, Tomato Juice, Salt, Chipotle Pepper, Methylcellulose, } \\
\text { Cooked Onion and Carrot Juice Concentrate, Jalapeno Pepper, Carrageen- } \\
\text { an, Garlic Powder, Natural Flavor, Paprika, Soy Sauce Powder (Soybeans, } \\
\text { Wheat, Salt), Gum Arabic, Vinegar, Citric Acid, Red Pepper, Green Pepper } \\
\text { Juice, Turmeric, Garlic Juice, Lime Juice. }\end{array}$ \\
\hline GP & Ground Pork at an $80 \%$ lean $20 \%$ fat ratio. \\
\hline GB & Ground Beef at an $80 \%$ lean $20 \%$ fat ratio. \\
\hline
\end{tabular}

Table 1: Ingredient list of two formulations of raw Beyond Meat Burger (BMB1 and BMB2), Impossible Foods Burger (IFB1 and IFB2), Black Bean Burger (BBB), 80/20 Ground Pork (GP), and 80/20 Ground Beef (GB).

All products were formed into $113 \mathrm{~g}(4 \mathrm{oz})$ patties and cooked on a pre-heated, non-stick anodized aluminum skillet to an internal temperature of $71^{\circ} \mathrm{C}$. After cooking, the product was placed on a stainless-steel rack to cool for $10 \mathrm{~min}$. Both uncooked and cooked patties were chilled at refrigerated temperatures $\left(0\right.$ to $\left.4^{\circ} \mathrm{C}\right)$ for 12 to $24 \mathrm{~h}$ prior to homogenization. Samples were then frozen in liquid nitrogen and immediately homogenized for $10 \mathrm{~s}$ on a low speed (1500rpm) and $30 \mathrm{~s}$ on a high speed (3500rpm) with a Robot Coupe BLITZER 6V (Robot Coupe USA Inc., Ridgeland, MS) blender, until a uniform powder was obtained. Homogenized samples of raw and cooked products were stored at $-80^{\circ} \mathrm{C}$ for further analysis. Unless otherwise indicated, analyses were performed at the Colorado State University (Fort Collins, $\mathrm{CO})$.

\section{Proximate and fiber analysis}

Moisture and ash: Moisture analysis was performed using the Association of Official Agricultural Chemists (AOAC) oven drying method 950.46 [22]. Approximately $1 \mathrm{~g}$ of samples were weighed into aluminum tins and allowed to dry for $24 \mathrm{~h}$ at $100^{\circ} \mathrm{C}$ in a forced air-drying oven. Percent moisture was calculated by determining the percent difference between wet and dry weight. Percent ash was determined using the ashing method described by 923.03 of the AOAC official methods [22]. Approximately $1 \mathrm{~g}$ of homogenate was placed into a pre-weighed crucible and set in a Thermolyne box furnace at $600^{\circ} \mathrm{C}$ for $18 \mathrm{~h}$. Percent ash was calculated by dividing the ash weight by the original wet sample weight and multiplying by 100 to obtain a percentage. All proximate data in the present work are reported on an as-received basis, as opposed to a dry-matter basis.

Crude fat and crude protein: Total lipid content was extracted using the method described by Folch et al., along with processes described in AOAC official method $983.23[23,24]$. Approximately $1 \mathrm{~g}$ of sample was homogenized in a 2:1 ratio of chloroform and methanol solution, respectively. Homogenized samples were placed onto an orbital shaker at room temperature for $20 \mathrm{~min}$, followed by filtering through ashless filter paper. Four $\mathrm{mL}$ of $0.9 \% \mathrm{NaCl}$ was added to the filtered sample, and the sample was placed in a refrigerator $\left(3 \pm 2^{\circ} \mathrm{C}\right)$ for $24 \mathrm{~h}$. When the filtrate separated into two phases, the lower phase was aspirated and placed into a pre-weighed scintillation vial. The liquid inside the vial was then dried under $\mathrm{N}_{2}$ gas. Following drying, the vial was allowed to air dry under a fume hood for $2 \mathrm{~h}$ and then placed into a forced air-drying oven to dry for $12 \mathrm{~h}$ at $100^{\circ} \mathrm{C}$. Percent fat was determined by dividing the fat weight by the original wet sample weight and multiplying by 100 to obtain a percentage.

Crude protein content was determined according to the AOAC method 992.15 x utilizing a TruSpec CN Carbon/Nitrogen Analyzer (Leco Corporation, St. Joseph, MI). Percent protein was calculated by multiplying the total percentage of nitrogen by a factor of 6.25 [24].

\section{Inductively Coupled Plasma (ICP) mass spectrometry analysis}

Minerals (Ca, Mg, K, Na, Fe, Zn, Cu, Mn, P) were analyzed by Eurofins Laboratories (Madison, WI) using theUSDA wet-ashing procedure and AOAC official methods 985.35, 984.27, 985.01 and 2011.14 $[24,25]$. Samples were digested in a microwave or on a hot plate with nitric acid, hydrochloric acid, or hydrogen peroxide. The amount of each element was determined with an ICP mass spectrophotometer (Agilent 8900, Santa Clara, CA) by comparing the emission of the unknown sample against emissions from standard solutions.

\section{Vitamin analysis}

Fat-soluble vitamins: Unless otherwise specified, vitamin analyses were performed at Eurofins Laboratories (Madison, WI). Vitamin A content was measured using HPLC methods described by Njeru et al. and Aloosilla et al. [26,27]. Vitamin D and 25-hydroxy-vitamin D analyses were performed using HPLC with UV detection according to AOAC method 2011.11 [25]. Vitamin E content was measured by Craft Technologies (Wilson, NC) using HPLC with a normal phase column as described by Speek et al. [28]. Ultraviolet detection with external calibration was performed as described by Cort et al. with an internal standard recovery as described by McMurray et al. post-analysis [29,30]. Vitamin K1 analysis was performed using AOAC official method 999.15, including HPLC and fluorescence detection [24].

Water-soluble vitamins: B-vitamins were analyzed according to the AOAC official methods utilized in the analysis of each vitamin was as follows: niacin AOAC 944.13 and 960.46; vitamin B-6 AOAC 961.15; riboflavin AOAC 960.46 and 940.33; thiamin AOAC 942.23, 953.17, and 957.17; pantothenic acid AOAC 945.74, 960.46, and 992.07 [24]. 


\section{Fatty acid and cholesterol analysis}

Total lipids were extracted from $1 \mathrm{~g}$ of the homogenized sample using methods from previously developed protocols [23,31]. Saponification and methylation of lipids were accomplished using the method described by Parks and Goins [32]. Individual lipids were separated via gas chromatography using a Hewlett Packard (Avondale, PA) Model 6890 series II gas chromatograph fixed with a series 7683 injector and flame ionization detector and fitted with a $100 \mathrm{~m} \times 0.25 \mathrm{~mm}$ fused silica capillary column (SP-2560 Supelco Inc. Bellefonte, PA).

Cholesterol content was analyzed at Eurofins Laboratories (Madison, WI) using AOAC official method 994.10 [22]. Samples were saponified using ethanolic potassium hydroxide. The unsaponifiable fraction that contained cholesterol and other sterols was extracted with toluene. Toluene was evaporated, and the residue was dissolved into dimethylformamide. Samples were derivatized to form trimethylsilyl ethers, and content was quantitatively determined by gas chromatography using 5 alpha-cholestenol as an internal standard.

\section{Amino acid analysis}

Amino acid profile was analyzed by Eurofins Laboratories (Madison, WI).Samples were hydrolyzed in $6 \mathrm{~N}$ hydrochloric acid for $24 \mathrm{~h}$ at approximately $110^{\circ} \mathrm{C}$. Phenol was added to the $6 \mathrm{~N}$ hydrochloric acid to prevent halogenation of tyrosine. Cystine and cysteine were converted to S-2-carboxyethylthiocysteine by the addition of dithiodipropionic acid, as described by Barkholt and Jensen [33]. Tryptophan was hydrolyzed from proteins by heating at approximately $110^{\circ} \mathrm{C}$ in $4.2 \mathrm{~N}$ sodium hydroxide as described by AOAC official method 988.15 [22]. Samples were analyzed by HPLC after pre-injection derivatization as described by Henderson et al. and Henderson and Brooks [34,35]. Primary amino acids were derivatized with o-phthalaldehyde and secondary amino acids were derivatized with fluorenylmethyl chloroformate before injection as described by Schuster [36]

\section{Statistical analysis}

Statistical analyses were performed using R software (v.3.6.1), whereby simple means and standard deviations for each nutrient component were obtained [37]. The Anova type III function from the Car package was used to determine statistical differences at an alpha level of 0.05 [38]. The emmeans function with a CLD display from the emmeans package was utilized to identify respective statistically significant differences [39]. Tukey adjusted pair wise comparisons were used for each test. Results for nutrient profiles of BMB1, BMB2, IFB1, IFB2, BBB, and GP were reported as least square means $(n=6)$ with standard deviation and a letter superscript designating statistical difference. Results for GB are reported as means with no standard deviation and no statistical superscript, as data was directly retrieved from USDA-ARS Food Data Central Database as a mean, with no standard deviations $[19,20]$. Finally, nutrient intakes per serving size were determined by multiplying the nutrient component mean by $113 \mathrm{~g} /$ serving.

\section{Results and Discussion}

\section{Proximate analysis}

Results from proximate analysis of raw and cooked samples are reported in tables 2 and 3, respectively. In general, crude protein and crude fat content did not differ $(P>0.05)$ for each PBMA and GP in raw and cooked states; although, GB contained the numerically greatest crude protein and crude fat content after cooking. These findings are important, validating that PBMAs are comparable to ABMs in crude protein and crude fat content.

\section{Mineral analysis}

Results from mineral analysis for previous and current formulations of raw and cooked samples are reported in tables 4 and 5, respectively. Calcium and sodium were considerably greater $(P<0.05)$ in PBMAs (BMB1, BMB2, IFB1, IFB2, and BBB) than in GP. One serving of cooked PBMA could supply between 3.0 to $24.4 \%$ of the adult (19 to 30 years of age) calcium Recommended Dietary Allowance (RDA) for males and females. The Dietary Guidelines for Americans suggest lowering dietary sodium intake, although one serving of PBMA may supply between 27.8 to $42.7 \%$ of the adult sodium RDA, and one serving of GB may supply approximately $22 \%$ of the adult RDA for sodium $[40,41]$. In general iron and zinc content was greater $(P<0.05)$ in most PBMAs than in GP, although zinc content was numerically greatest in GB than in the PBMAs and GP. One serving of cooked PBMA may supply between 28.8 to $83.7 \%$ and 15.3 to 44.7 $\%$ of the iron RDA for adult males and females, respectively [40]. Nonetheless, iron found in plant sources is exclusively non-heme iron, which is less bio available than heme iron found in GP and GB [42].

While these minerals are nutritionally essential, the presence of phytates, fibrous plant materials, mineral antagonists, and the incorporation of minerals in the food matrix may inhibit absorption [43-46]. High calcium and magnesium levels have been known to decrease iron and potassium absorption, while high iron concentrations have been known to contribute to lower manganese absorption [47-49]. While it has been demonstrated that calcium doses (similar to those present in IFB1) do not significantly reduce iron absorption, calcium has been known to inhibit iron absorption when minerals were ingested from the same food, which could be the case in PBMAs. Additionally, high sodium and potassium levels may increase urinary mineral losses [48-51].

\section{Vitamin analysis}

Results from vitamin analysis for previous and current formulations of raw and cooked samples are reported in tables 6 and 7, respectively. Fat-soluble vitamin $\mathrm{A}, \mathrm{D}_{2}$, and $\mathrm{D}_{3}$ were found to be below the detection limits $(<0.3 \mathrm{mcg} / \mathrm{g}$ for vitamin $\mathrm{A}$, and $<0.001 \mathrm{mcg} / \mathrm{g}$ for vitamin $\mathrm{D}_{2}$ and $\mathrm{D}_{3}$ ) in all raw and cooked products, with only vitamin $\mathrm{K}$, beingdetected in $\mathrm{BMB} 1, \mathrm{BMB} 2$, and $\mathrm{BBB}$ samples at concentrations slightly above the detection limit. Vitamin $\mathrm{E}$ content in raw and cooked PBMAs was substantially greater $(P<0.05)$ than in raw and cooked GP. Vitamin E content in GB can be variable depending on the production and processing parameters, although GB was numerically less than the PBMAs and GP in the samples tested for this study [52]. One serving of cooked PBMA may supply between 14.7 to $60.1 \%$ of the RDA for adults (19-30y) [40]. Generally, vitamin E supplementation in foods contributes a significant portion (>50\% RDA) to the American diet, but absorption of vitamin $\mathrm{E}$ in the human intestine is highly variable and can be impacted by the amount consumed, fat content, food matrix, and the presence of other fat-soluble nutrients $[53,54]$

For each B-vitamin, IFB1 and IFB2 were statistically greater $(P<0.05)$ than or numerically comparable to GP, excluding niacin $\left(\mathrm{B}_{3}\right)$ and pantothenic acid $\left(\mathrm{B}_{5}\right)$, for which GP was greater $(P<0.05)$. One serving of IFB1 or IFB2 would surpass the adult thiamin $\left(B_{1}\right)$ RDA 
Citation: Swing CJ, Thompson TW, Guimaraes O, Geornaras I, Engle TE, et al. (2021) Nutritional Composition of Novel Plant-Based Meat Alternatives and Traditional Animal-Based Meats. J Food Sci Nutr 7: 109.

- Page 4 of 11 •

\begin{tabular}{|c|c|c|c|c|c|c|c|}
\hline Component & BMB1 & BMB2 & IFB1 & IFB2 & BBB & GP & GB* \\
\hline Dry Matter & $32.1 \pm 0.8^{c}$ & $42.9 \pm 0.8^{\mathrm{a}}$ & $37.2 \pm 2.0^{\mathrm{b}}$ & $42.3 \pm 0.4^{\mathrm{a}}$ & $32.5 \pm 2.7^{\mathrm{c}}$ & $37.1 \pm 1.3^{\mathrm{b}}$ & 38.0 \\
\hline Moisture & $67.9 \pm 0.8^{\mathrm{a}}$ & $57.1 \pm 0.8^{c}$ & $62.8 \pm 2.0^{\mathrm{b}}$ & $57.7 \pm 0.4^{\mathrm{c}}$ & $67.5 \pm 2.7^{\mathrm{a}}$ & $62.9 \pm 1.3^{\mathrm{b}}$ & 62.0 \\
\hline Ash & $1.5 \pm 0.7^{\mathrm{b}}$ & $1.7 \pm 0.2^{\mathrm{b}}$ & $1.5 \pm 0.5^{\mathrm{b}}$ & $2.5 \pm 0.2^{\mathrm{a}}$ & $1.3 \pm 0.4^{\mathrm{b}}$ & $1.8 \pm 0.4^{\mathrm{ab}}$ & 0.84 \\
\hline Crude Fat & $10.8 \pm 3.8^{\mathrm{a}}$ & $13.1 \pm 2.2^{\mathrm{a}}$ & $12.0 \pm 5.0^{\mathrm{a}}$ & $11.7 \pm 1.9^{\mathrm{a}}$ & $10.8 \pm 3.8^{\mathrm{a}}$ & $10.2 \pm 4.7^{\mathrm{a}}$ & 20.0 \\
\hline Crude Protein & $20.0 \pm 3.4^{\mathrm{ab}}$ & $18.6 \pm 0.9^{\mathrm{ab}}$ & $22.0 \pm 4.1^{\mathrm{ab}}$ & $17.2 \pm 0.9^{\mathrm{b}}$ & $24.0 \pm 6.5^{\mathrm{a}}$ & $22.6 \pm 3.2^{\mathrm{ab}}$ & 17.0 \\
\hline
\end{tabular}

Table 2: Proximate analysis (percent \pm SD) of two formulations of raw Beyond Meat Burger (BMB1 and BMB2), Impossible Foods Burger (IFB1 and IFB2), Black Bean Burger (BBB), 80/20 Ground Pork (GP), and 80/20 Ground Beef (GB).

Note: ${ }^{\text {a-b }}$ Means within a row with different subscripts differ statistically $(\mathrm{P}<0.05)$.

*Data collected from USDA nutrient database [19].

\begin{tabular}{|c|c|c|c|c|c|c|c|}
\hline Component & BMB1 & BMB2 & IFB1 & IFB2 & BBB & GP & GB* \\
\hline Dry Matter & $36.7 \pm 3.5^{\mathrm{c}}$ & $50.3 \pm 0.9^{\mathrm{a}}$ & $45.0 \pm 0.7^{\mathrm{b}}$ & $47.7 \pm 1.0^{\mathrm{ab}}$ & $30.7 \pm 2.6^{d}$ & $44.8 \pm 2.2^{\mathrm{b}}$ & 44.0 \\
\hline Moisture & $63.3 \pm 3.5^{\mathrm{b}}$ & $49.7 \pm 0.9^{\mathrm{d}}$ & $55.0 \pm 0.7^{\mathrm{c}}$ & $52.3 \pm 1.0^{\mathrm{cd}}$ & $69.3 \pm 2.6^{\mathrm{a}}$ & $55.2 \pm 2.2^{\mathrm{c}}$ & 56.0 \\
\hline Ash & $1.8 \pm 0.6^{\mathrm{bc}}$ & $2.1 \pm 0.2^{\mathrm{b}}$ & $1.6 \pm 0.2^{\mathrm{bc}}$ & $3.0 \pm 0.2^{\mathrm{a}}$ & $1.5 \pm 0.4^{\mathrm{bc}}$ & $1.3 \pm 0.4^{\mathrm{c}}$ & 1.0 \\
\hline Crude Fat & $11.9 \pm 5.2^{\mathrm{a}}$ & $11.6 \pm 3.9^{\mathrm{a}}$ & $9.2 \pm 3.1^{\mathrm{a}}$ & $11.2 \pm 1.7^{\mathrm{a}}$ & $11.9 \pm 4.3^{\mathrm{a}}$ & $11.1 \pm 5.9^{\mathrm{a}}$ & 18.0 \\
\hline Crude Protein & $23.3 \pm 4.4^{\mathrm{a}}$ & $23.8 \pm 1.5^{\mathrm{a}}$ & $20.3 \pm 3.6^{\mathrm{a}}$ & $20.2 \pm 0.5^{\mathrm{a}}$ & $22.4 \pm 4.9^{\mathrm{a}}$ & $21.5 \pm 3.0^{\mathrm{a}}$ & 26.0 \\
\hline
\end{tabular}

Table 3: Proximate analysis (percent \pm SD) of two formulations of cooked Beyond Meat Burger (BMB1 and BMB2), Impossible Foods Burger (IFB1 and IFB2), Black Bean Burger (BBB), 80/20 Ground Pork (GP), and 80/20 Ground Beef (GB).

Note: ${ }^{\text {a-c }}$ Means within a row with different subscripts differ statistically $(\mathrm{P}<0.05)$

*Data collected from USDA nutrient database [20].

\begin{tabular}{|c|c|c|c|c|c|c|c|}
\hline Component & BMB1 & BMB2 & IFB1 & IFB2 & BBB & GP & GB* \\
\hline Calcium & $213.8 \pm 11.6 \mathrm{c}$ & $819.8 \pm 74.0 \mathrm{~b}$ & $257.5 \pm 6.7 \mathrm{c}$ & $1860 \pm 46.5 \mathrm{a}$ & $881.8 \pm 40.5 \mathrm{~b}$ & $105.8 \pm 61.9 \mathrm{~d}$ & 180 \\
\hline Magnesium & $190.8 \pm 7.8 \mathrm{c}$ & $350 \pm 30.1 \mathrm{~b}$ & $120.7 \pm 7.2 \mathrm{~d}$ & $714 \pm 19.3 \mathrm{a}$ & $367.3 \pm 9.0 \mathrm{~b}$ & $178.5 \pm 16.3 \mathrm{c}$ & 170 \\
\hline Phosphorus & $1888.3 \pm 74.4 \mathrm{~b}$ & $2423.3 \pm 160.7 \mathrm{a}$ & $1296.7 \pm 28.8 \mathrm{~d}$ & $1840 \pm 54.7 \mathrm{~b}$ & $1263.3 \pm 48.4 \mathrm{~d}$ & $1596.7 \pm 126.6 \mathrm{c}$ & 1580 \\
\hline Potassium & $2828.3 \pm 188.1 \mathrm{bc}$ & $2431.7 \pm 247.9 \mathrm{c}$ & $3096.7 \pm 89.4 \mathrm{~b}$ & $5760 \pm 197.9 \mathrm{a}$ & $2930 \pm 129.6 \mathrm{bc}$ & $3176.7 \pm 702.7 \mathrm{~b}$ & 2700 \\
\hline Sodium & $3328.3 \pm 205.5 \mathrm{~b}$ & $3230.0 \pm 275.3 \mathrm{~b}$ & $4935 \pm 167.0 \mathrm{a}$ & $3608.3 \pm 203.9 \mathrm{~b}$ & $3273.3 \pm 191.0 \mathrm{~b}$ & $995.5 \pm 1281.4 \mathrm{c}$ & 660 \\
\hline Copper & $3.4 \pm 0.4 \mathrm{a}$ & $2.1 \pm 0.3 \mathrm{~b}$ & $3.8 \pm 0.4 \mathrm{a}$ & $2.7 \pm 0.5 \mathrm{~b}$ & $2.42 \pm 0.16 \mathrm{~b}$ & $0.7 \pm 0.1 \mathrm{c}$ & 0.61 \\
\hline Iron & $43.4 \pm 2.9 \mathrm{a}$ & $36.6 \pm 2.6 \mathrm{~b}$ & $22.3 \pm 0.9 \mathrm{c}$ & $36.3 \pm 3.6 \mathrm{~b}$ & $17.62 \pm 0.69 \mathrm{~d}$ & $7.91 \pm 2.3 \mathrm{e}$ & 19.4 \\
\hline Manganese & $2.5 \pm 0.5 \mathrm{~d}$ & $6.9 \pm 0.8 \mathrm{~b}$ & $4.4 \pm 0.3 \mathrm{c}$ & $10.3 \pm 0.7 \mathrm{a}$ & $4.92 \pm 0.28 \mathrm{c}$ & $0.18 \pm 0 \mathrm{e}$ & 0.10 \\
\hline Zinc & $20.9 \pm 1.3 \mathrm{c}$ & $23.3 \pm 2.7 \mathrm{c}$ & $29.7 \pm 1.4 \mathrm{~b}$ & $48.3 \pm 2.5 \mathrm{a}$ & $8.72 \pm 0.75 \mathrm{~d}$ & $20.8 \pm 5.0 \mathrm{c}$ & 41.8 \\
\hline
\end{tabular}

Table 4: Mineral composition (ppm \pm SD) of two formulations of raw Beyond Meat Burger (BMB1 and BMB2), Impossible Foods Burger (IFB1 and IFB2), Black Bean Burger (BBB), 80/20 Ground Pork (GP), and 80/20 Ground Beef (GB).

Note: ${ }^{\text {a-e }}$ Means within a row with different subscripts differ statistically $(\mathrm{P}<0.05)$.

*Data collected from USDA nutrient database [19].

\begin{tabular}{|c|c|c|c|c|c|c|c|}
\hline Component & BMB1 & BMB2 & IFB1 & IFB2 & BBB & GP & GB* \\
\hline Calcium & $267.3 \pm 15.2^{\mathrm{c}}$ & $1068.2 \pm 89.3^{\mathrm{b}}$ & $297.0 \pm 15.2^{\mathrm{c}}$ & $2165.0 \pm 69.5^{\mathrm{ab}}$ & $1004.5 \pm 50.4^{b}$ & $139.1 \pm 87.3^{\mathrm{d}}$ & 240.0 \\
\hline Magnesium & $235.3 \pm 6.3^{\mathrm{d}}$ & $446.7 \pm 31.7^{b}$ & $140.8 \pm 6.5^{\mathrm{e}}$ & $827.0 \pm 23.1^{\mathrm{a}}$ & $410.0 \pm 9.6^{\mathrm{c}}$ & $239.2 \pm 17.7^{\mathrm{d}}$ & 200.0 \\
\hline Phosphorus & $2315.0 \pm 79.4^{b}$ & $3120.0 \pm 182.5^{\mathrm{a}}$ & $1513.3 \pm 28.8^{\mathrm{c}}$ & $2143.3 \pm 60.2^{\mathrm{b}}$ & $1425.0 \pm 47.2^{\mathrm{c}}$ & $2146.7 \pm 121.8^{\mathrm{b}}$ & 1940.0 \\
\hline Potassium & $3378.3 \pm 367.1^{\mathrm{c}}$ & $3056.7 \pm 287.3^{c}$ & $3590.0 \pm 61.0^{\mathrm{bc}}$ & $6753.3 \pm 326.2^{\mathrm{a}}$ & $3270.0 \pm 120.7^{\mathrm{c}}$ & $4220.0 \pm 834.9^{\mathrm{b}}$ & 3040.0 \\
\hline Sodium & $4135.0 \pm 355.8^{\mathrm{b}}$ & $4186.7 \pm 262.0^{\mathrm{b}}$ & $5666.7 \pm 213.0^{\mathrm{a}}$ & $4240.0 \pm 219.3^{b}$ & $3690.0 \pm 216.4^{b}$ & $1277.8 \pm 1586.4^{c}$ & 750 \\
\hline Iron & $60.0 \pm 5.5^{\mathrm{a}}$ & $47.4 \pm 3.0^{\mathrm{b}}$ & $27.0 \pm 1.4^{\mathrm{c}}$ & $42.6 \pm 3.2^{\mathrm{b}}$ & $20.7 \pm 1.0^{\mathrm{d}}$ & $10.8 \pm 2.6^{\mathrm{e}}$ & 24.8 \\
\hline Manganese & $3.0 \pm 0.3^{\mathrm{d}}$ & $9.0 \pm 0.8^{\mathrm{b}}$ & $5.0 \pm 0.4^{\mathrm{c}}$ & $12.0 \pm 0.8^{\mathrm{a}}$ & $5.5 \pm 0.3^{\mathrm{c}}$ & $0.2 \pm 0^{\mathrm{e}}$ & 0.11 \\
\hline Zinc & $25.6 \pm 2.0^{\mathrm{c}}$ & $30.3 \pm 2.8^{\mathrm{bc}}$ & $34.2 \pm 1.8^{\mathrm{b}}$ & $56.8 \pm 3.4^{\mathrm{a}}$ & $9.9 \pm 0.9^{\mathrm{d}}$ & $28.2 \pm 5.5^{\mathrm{c}}$ & 62.5 \\
\hline
\end{tabular}

Table 5: Mineral composition (ppm \pm SD) of two formulations of cooked Beyond Meat Burger (BMB1 and BMB2), Impossible Foods Burger (IFB1 and IFB2), Black Bean Burger (BBB), 80/20 Ground Pork (GP), and 80/20 Ground Beef (GB).

Note: ${ }^{\text {aee }}$ Means within a row with different subscripts differ statistically $(\mathrm{P}<0.05)$.

*Data collected from USDA nutrient database [20]. 
Citation: Swing CJ, Thompson TW, Guimaraes O, Geornaras I, Engle TE, et al. (2021) Nutritional Composition of Novel Plant-Based Meat Alternatives and Traditional Animal-Based Meats. J Food Sci Nutr 7: 109.

- Page 5 of 11 •

\begin{tabular}{|c|c|c|c|c|c|c|c|}
\hline Component & BMB1 & BMB2 & IFB1 & IFB2 & BBB & GP & GB* \\
\hline Vitamin A & $<0.3$ & $<0.3$ & $<0.3$ & $<0.3$ & $<0.3$ & $<0.3$ & 0.04 \\
\hline Vitamin $\mathrm{D}_{2}$ & $<0.001$ & $<0.001$ & $<0.001$ & $<0.001$ & $<0.001$ & $<0.001$ & 0.07 \\
\hline Vitamin $\mathrm{D}_{3}$ & $<0.001$ & $<0.001$ & $<0.001$ & $<0.001$ & $<0.001$ & $<0.001$ & NA \\
\hline Vitamin E & $21.7 \pm 4.5^{\mathrm{c}}$ & $17.1 \pm 3.4^{\mathrm{c}}$ & $33.9 \pm 5.9^{b}$ & $71.0 \pm 4.9^{\mathrm{a}}$ & $15.9 \pm 1.3^{\mathrm{c}}$ & $5.1 \pm 0.2^{\mathrm{d}}$ & 1.7 \\
\hline Vitamin $\mathrm{K}_{1}$ & $0.2 \pm 0.02^{\mathrm{a}}$ & $0.1 \pm 0.03^{\mathrm{b}}$ & $<0.04$ & $<0.04$ & $0.06 \pm 0.01^{\mathrm{c}}$ & 0.04 & 0.02 \\
\hline Thiamin $\left(\mathrm{B}_{1}\right)$ & $0.6 \pm 0.5^{b}$ & $0.5 \pm 0.1^{\mathrm{b}}$ & $182.3 \pm 7.5^{\mathrm{a}}$ & $190.5 \pm 19.5^{\mathrm{a}}$ & $1.1 \pm 1.1^{\mathrm{b}}$ & $3.3 \pm 1.0^{\mathrm{b}}$ & 0.43 \\
\hline Riboflavin $\left(\mathrm{B}_{2}\right)$ & $1.2 \pm 0.1^{\mathrm{c}}$ & $1.1 \pm 0.2^{\mathrm{c}}$ & $3.8 \pm 0.3^{\mathrm{a}}$ & $2.9 \pm 0.2^{\mathrm{b}}$ & $2.5 \pm 0.3^{\mathrm{b}}$ & $2.5 \pm 0.5^{\mathrm{b}}$ & 1.51 \\
\hline $\operatorname{Niacin}\left(\mathrm{B}_{3}\right)$ & $3.5 \pm 0.3^{\mathrm{b}}$ & $5.6 \pm 0.4^{b}$ & $52.68 \pm 4.9^{\mathrm{a}}$ & $51.7 \pm 4.9^{\mathrm{a}}$ & $8.4 \pm 0.4^{\mathrm{b}}$ & $56.0 \pm 13.7^{\mathrm{a}}$ & 42.3 \\
\hline Pantothenic Acid $\left(\mathrm{B}_{5}\right)$ & $3.6 \pm 0.3^{b}$ & $1.8 \pm 0.2^{\mathrm{d}}$ & $3.4 \pm 0.3^{\text {bc }}$ & $2.0 \pm 0.2^{\mathrm{cd}}$ & $3.7 \pm 0.3^{b}$ & $8.1 \pm 2.0^{\mathrm{a}}$ & 5.0 \\
\hline Pyridoxine Free Base $\left(\mathrm{B}_{6}\right)$ & $0.4 \pm 0.1^{\mathrm{c}}$ & $0.5 \pm 0.1^{\mathrm{c}}$ & $2.9 \pm 0.2^{\mathrm{b}}$ & $4.9 \pm 0.2^{\mathrm{a}}$ & $1.0 \pm 0.1^{\mathrm{c}}$ & $3.6 \pm 1.2^{\mathrm{b}}$ & 3.2 \\
\hline Biotin $\left(\mathrm{B}_{7}\right)$ & $0.06 \pm 0.01^{b}$ & $0.05 \pm 0.0^{\mathrm{bc}}$ & $0.04 \pm 0.01^{\mathrm{c}}$ & $0.1 \pm 0.02^{\mathrm{a}}$ & $0.05 \pm 0.01^{\mathrm{bc}}$ & $0.04 \pm 0.01^{\mathrm{c}}$ & NA \\
\hline Folates $\left(\mathrm{B}_{9}\right)$ & NT & $0.3 \pm 0.04^{\mathrm{b}}$ & NT & $1.1 \pm 0.1^{\mathrm{a}}$ & NT & $0.05^{* *}$ & 0.1 \\
\hline
\end{tabular}

Table 6: Vitamin composition ( $\mathrm{mcg} / \mathrm{g} \pm \mathrm{SD}$ ) of two formulations of raw Beyond Meat Burger (BMB1 and BMB2), Impossible Foods Burger (IFB1 and IFB2), Black Bean Burger (BBB), 80/20 Ground Pork (GP), and 80/20 Ground Beef (GB).

Note: ${ }^{\text {a-d }}$ Means within a row with different subscripts differ statistically $(\mathrm{P}<0.05)$.

*Data collected from USDA nutrient database [19]

NA: Not Applicable; NT: Not tested.

$<$ : Sample levels were not identified above the analysis thresholds for detection

\begin{tabular}{|c|c|c|c|c|c|c|c|}
\hline Component & BMB1 & BMB2 & IFB1 & IFB2 & BBB & GP & GB* \\
\hline Vitamin A & $<0.3$ & $<0.3$ & $<0.3$ & $<0.3$ & $<0.3$ & $<0.3$ & 0.03 \\
\hline Vitamin $\mathrm{D}_{2}$ & $<0.001$ & $<0.001$ & $<0.001$ & $<0.001$ & $<0.001$ & $<0.001$ & 0.05 \\
\hline Vitamin $\mathrm{D}_{3}$ & $<0.001$ & $<0.001$ & $<0.001$ & $<0.001$ & $<0.001$ & $<0.001$ & NA \\
\hline Vitamin E & $26.6 \pm 6.7^{\mathrm{c}}$ & $18.4 \pm 4.3^{\mathrm{d}}$ & $38.8 \pm 2.1^{\mathrm{b}}$ & $80.3 \pm 4.8^{\mathrm{a}}$ & $19.6 \pm 0.9^{\mathrm{cd}}$ & $5.74 \pm 1.8^{\mathrm{e}}$ & 1.2 \\
\hline Vitamin $\mathrm{K}_{1}$ & $0.3 \pm 0.03^{\mathrm{a}}$ & $0.2 \pm 0.03^{\mathrm{b}}$ & $<0.04$ & $<0.04$ & $0.06 \pm 0.01^{\mathrm{c}}$ & $<0.04$ & 0.02 \\
\hline Thiamin $\left(\mathrm{B}_{1}\right)$ & $0.3 \pm 0.1^{\mathrm{b}}$ & $0.7 \pm 0.1^{\mathrm{b}}$ & $197.3 \pm 9.7^{\mathrm{a}}$ & $206.5 \pm 13.3^{\mathrm{a}}$ & $0.7 \pm 0.04^{\mathrm{b}}$ & $4.0 \pm 1.3^{b}$ & 0.5 \\
\hline Riboflavin $\left(\mathrm{B}_{2}\right)$ & $1.6 \pm 0.2^{\mathrm{c}}$ & $1.5 \pm 0.1^{\mathrm{c}}$ & $4.4 \pm 0.2^{\mathrm{a}}$ & $3.2 \pm 0.2^{\mathrm{b}}$ & $3.0 \pm 0.2^{\mathrm{b}}$ & $3.1 \pm 0.4^{\mathrm{b}}$ & 1.8 \\
\hline $\operatorname{Niacin}\left(\mathrm{B}_{3}\right)$ & $4.3 \pm 0.3^{\mathrm{d}}$ & $6.0 \pm 0.5^{\text {cd }}$ & $62.2 \pm 3.0^{\mathrm{b}}$ & $58.2 \pm 2.9^{\mathrm{b}}$ & $10.5 \pm 0.6^{\mathrm{c}}$ & $80.0 \pm 6.9^{\mathrm{a}}$ & 51.0 \\
\hline Pantothenic Acid $\left(\mathrm{B}_{5}\right)$ & $4.0 \pm 0.2^{\mathrm{b}}$ & $3.0 \pm 0.4^{\mathrm{cd}}$ & $3.5 \pm 0.2^{\mathrm{bc}}$ & $2.1 \pm 0.2^{\mathrm{d}}$ & $3.9 \pm 0.3^{\mathrm{bc}}$ & $8.9 \pm 1.3^{\mathrm{a}}$ & 6.6 \\
\hline Pyridoxine Free Base $\left(\mathrm{B}_{6}\right)$ & $0.5 \pm 0.1^{\mathrm{d}}$ & $0.5 \pm 0.1^{\mathrm{d}}$ & $3.1 \pm 0.2^{\mathrm{b}}$ & $5.6 \pm 0.5^{\mathrm{a}}$ & $1.2 \pm 0.1^{\mathrm{c}}$ & $3.0 \pm 0.5^{\mathrm{b}}$ & 3.7 \\
\hline Biotin $\left(\mathrm{B}_{7}\right)$ & $0.1 \pm 0.01^{\mathrm{b}}$ & $0.1 \pm 0^{\mathrm{b}}$ & $0.1 \pm 0.01^{\mathrm{c}}$ & $0.2 \pm 0.01^{\mathrm{a}}$ & $0.07 \pm 0.01^{\mathrm{b}}$ & $0.05 \pm 0.01^{\mathrm{c}}$ & $\mathrm{NA}$ \\
\hline
\end{tabular}

Table 7: Vitamin composition ( $\mathrm{mcg} / \mathrm{g} \pm \mathrm{SD}$ ) of two formulations of cooked Beyond Meat Burger (BMB1 and BMB2), Impossible Foods Burger (IFB1 and IFB2), Black Bean Burger (BBB), 80/20 Ground Pork (GP), and 80/20 Ground Beef (GB).

Note: ${ }^{\text {a-e }}$ Means within a row with different subscripts differ statistically $(\mathrm{P}<0.05)$.

*Data collected from USDA nutrient database [20].

NA: Not Applicable; NT: Not tested.

$<$ : Sample levels were not identified above the analysis thresholds for detection.

for males and females at 1.2 and $1.1 \mathrm{mg} / \mathrm{d}$, respectively; although one serving of GP would also meet the thiamin $\left(\mathrm{B}_{1}\right)$ RDA for adults as well. Furthermore, one serving of PBMA would supply between 41.2 to $43.8 \%$ of the adult RDA for niacin $\left(B_{3}\right)$, while one serving of cooked GP or GB would supply approximately 36.2 to $56.3 \%$ of the adult RDA for niacin ( $\mathrm{B}_{3}$ ) RDA [40]. One serving of cooked GP or GB would supply approximately 14.9 to $20.0 \%$ of the adult RDA for pantothenic acid $\left(\mathrm{B}_{5}\right)$, while PBMAs would supply between 4.8 to $9.1 \%$ of the adult RDA for pantothenic acid $\left(\mathrm{B}_{5}\right)$ [40]. Finally, cooked BMB2 and IFB2 would supply approximately 11.6 to $32.1 \%$ of the adult RDA for folate $\left(\mathrm{B}_{9}\right)$, while cooked $\mathrm{GB}$ would supply approximately $2.8 \%$ of the adult RDA forfolate $\left(\mathrm{B}_{9}\right)[40]$.
While research has demonstrated that some B-vitamins may have poor thermo stability, photo stability, and evaporation loss during storage and cooking, the fluorometric and microbial analysis performed in this study did not demonstrate high B-vitamin loss [55-58]. Nonetheless, the bioavailability of B-vitamins can be impacted by multiple factors [59]. Niacin $\left(B_{3}\right)$ is usually chemically bound when found in plant materials, while thiamin $\left(\mathrm{B}_{1}\right)$ and pyridoxine $\left(\mathrm{B}_{6}\right)$ can undergo Maillard reactions which may affect bioavailability $[60,61]$. Moreover, the crystalline nature of thiamin $\left(\mathrm{B}_{1}\right)$ supplements and the presence of other minerals may impact bioavailability [62]. Considering the high concentrations of thiamin $\left(\mathrm{B}_{1}\right)$ and pyridoxine $\left(\mathrm{B}_{6}\right)$ in IFB1 and IFB2 products, cooking may reduce the availability of these 
vitamins through the Maillard reaction [63]. Furthermore, high thiamin $\left(\mathrm{B}_{1}\right)$ concentrations may inhibit riboflavin $\left(\mathrm{B}_{2}\right)$ and pyridoxine $\left(\mathrm{B}_{6}\right)$ absorption $[64,65]$. Copper, calcium, iron, and other minerals, in high concentrations may also antagonize B-vitamin absorption, although the effect of other antagonists, food matrix, and other factors impacting B-vitamin absorption would have to be researched [66].

\section{Fatty acid analysis}

Results from fatty acid analysis for previous and current formulations of raw and cooked samples are reported in tables 8 and 9, respectively. Raw and cooked PBMAs were below the detection limit $(<0.01 \mathrm{mg} / \mathrm{g})$ for cholesterol levels, while cooked GP contained approximately $0.86 \mathrm{mg} / \mathrm{g}$. A major finding of the fatty acid analysis was that the total saturated fatty acid content of PBMAs ranged between 43 and $44 \%$ for BMB2 and IFB2, while GP and GB ranged between 39 and $42 \%$ of the total fatty acids. Additionally, total monounsaturated fatty acid content of BMB2 and IFB2 was similar to GB (at less than $55 \%$ ), whereas GP contained the most total polyunsaturated fatty acid content (approximately 24\%). As previously mentioned, not only did crude fat content not differ $(P>0.05)$ between PBMAs and GP, but total saturated fatty acid content is in BMB2 and IFB2 was similar to GP and GB. Furthermore, raw and cooked GP and GB had higher numerical values for total mono-unsaturated and polyunsaturated fatty acids than BMB2 and IFB2.

Palmitic (C16:0) and stearic (C18:0) acid compositions were greater $(P<0.05)$ in GP than in the PBMAs, although, oleic $(\mathrm{C} 18: 1)$ acid content in BMB2 and IFB2 was greater $(P<0.05)$ than GP. Substantial increases $(P<0.05)$ in oleic $(\mathrm{C} 18: 1)$ and linoleic $(\mathrm{C} 18: 2)$ acid content from IFB1 to IFB2 may be a result of sunflower oil which was used in addition to coconut oil in the IFB2 formulation [67]. Cocoa butter was substituted in the BMB2 formulation for sunflower oil, which may explain the increase $(P<0.05)$ in oleic acid $(\mathrm{C} 18: 1)$ content from BMB1 and decrease $(P<0.05)$ in linoleic acid $(\mathrm{C} 18: 2)$ content from BMB1 [67,68].

Linoleic (C18:2) and a-linolenic (C18:3) acids, cannot be synthesized de novo and are essential for human growth and development [69].The BMB1 and BBB products proved to be a potentially excellent sources of these essential fatty acids compared to the other PBMAs. Conversely, GP was an excellent source of linoleic (C18:2) and arachidonic (C20:4) acid compared to BMB2 and IFB2. Fatty acids are readily absorbed in the human body, but the presence of fibrous materials and various thickeners and binders incorporated into PBMA formulations may inhibit fatty acid absorption $[70,71]$.

\section{Amino acid analysis}

Results from amino acid analysis for previous and current formulations of raw and cooked samples are reported in tables 10 and 11, respectively. Total essential amino acid content of raw BMB1, BMB2, and IFB1 ranged between 70 and $76 \mathrm{mg} / \mathrm{g}$ while total essential amino acid content of raw GP and GB ranged between 66 and $68 \mathrm{mg} / \mathrm{g}$. Histidine, methionine, and lysine concentrations were greater $(P<0.05)$ in GP compared to PBMAs. Conversely, GB was numerically greater than the PBMAs and GP in histidine, methionine, and lysine content, which may confer preferable anabolic capacity for the ABMs compared to the PBMAs [72]. Cooked BMB2 was greater $(P<0.05)$ than GP in isoleucine and phenylalanine content, probably as pea and mung beanprotein isolates (used in BMB2 formulation) are excellent sources of these essential amino acids [73,74]. Finally, IFB2 and BBB was either numerically or statistically less than $(P<0.05)$ GP in each essential amino acid; and were also less than $(P<0.05) \mathrm{BMB} 2$ in each essential amino acid assessed, except threonine and tryptophan.

The PBMAs were either numerically comparable to or statistically greater than $(P<0.05)$ ABMs for most non-essential amino acid profiles. BMB2 was greater than $(P<0.05) \mathrm{G}$ Pin arginine, aspartic acid, glutamic acid, serine, and tyrosine content. Conversely, GB was numerically greater than the PBMAs and GP in glycine and alanine content. Substantial increases $(P<0.05)$ in glutamic acid, glycine, serine, and proline were observed from BMB1 to BMB2 formulation, probably due to the inclusion of and mung bean protein isolates which are relatively high in the aforementioned amino acids [74,75]. In contrast, IFB2 and BBB were either numerically comparable to or statistically less $(P<0.05)$ than GP and BMB2 in most non-essential amino acids assessed. Substantial decreases $(P<0.05)$ in nearly each non-essential amino acid were observed from IFB1 to IFB2, as wheat protein isolates are exceptionally high in glutamic acid, proline, and potato protein is high in many of the other non-essential amino acids [72].

The presence of fibrous material and anti nutrient components in some plant products may inhibit protein digestibility of PBMAs [76]. The Protein Digestibility-Corrected Amino Acid Score (PDCAAS) is a simple evaluation of protein quality related to the amount of the first limiting amino acid in a test protein to the human metabolic requirement of that corresponding amino acid. The PDCAAS of soy, wheat, pea, mung bean, and bean proteins are 95, 96, 88, 76, and $78 \%$, respectively, while the PDCAAs score for pork and beef has a score of $100 \%$ [77-79]. However, it has been suggested that PDCAAS digestibility scores fail to account for anti nutrient factors present in plant materials and substantially over-estimate the digestibility of many plant proteins [80]. Plant-based proteins have been reported to have depressed bioavailability compared to ABMs, although the absorption of amino acids from PBMAs in comparison to ABMs should be further evaluated [81].

\section{Conclusion}

The PBMAs were numerically comparable to GP in crude protein and crude fat content. In general, the PBMAs were high in several minerals, potentially competing for absorption, although this effect would have to be further researched. The IFB2 was high in thiamin $\left(\mathrm{B}_{1}\right)$, niacin $\left(\mathrm{B}_{3}\right)$, pyridoxine $\left(\mathrm{B}_{6}\right)$, biotin $\left(\mathrm{B}_{7}\right)$, and folates $\left(\mathrm{B}_{9}\right)$. However, high thiamin $\left(\mathrm{B}_{1}\right)$ concentrations may inhibit the absorption of riboflavin $\left(\mathrm{B}_{2}\right)$ and pyridoxine $\left(\mathrm{B}_{6}\right)$. Crude fat and total saturated fatty acid content were similar between BMB2, IFB2, and GP. In particular, BMB2 and IFB2 more closely resembled the fatty acid profile of GP, than the previous formulations of BMB1 and IFB1. The PBMAs were generally comparable to GP in most essential amino acid profiles, although high lysine and methionine content. Overall, PBMAs were generally comparable to ABMs in many of the nutrient profiles assessed. However, it is important to investigate further the bioavailability of these nutrients, when incorporated into a PBMA food matrix and exposed to different anti nutrient compounds and antagonistic interactions.

\section{Acknowledgement}

Funding for this research was provided in part by The Beef Checkoff, Denver, $\mathrm{CO}$, and in part by the National Pork Checkeff fliye Iô 
Citation: Swing CJ, Thompson TW, Guimaraes O, Geornaras I, Engle TE, et al. (2021) Nutritional Composition of Novel Plant-Based Meat Alternatives and Traditional Animal-Based Meats. J Food Sci Nutr 7: 109.

- Page 7 of $11 \bullet$

\begin{tabular}{|c|c|c|c|c|c|c|c|}
\hline Component & BMB1 & BMB2 & IFB1 & IFB2 & BBB & GP & GB* \\
\hline Cholesterol & $<0.01 \pm 0^{\mathrm{b}}$ & $<0.01 \pm 0^{\mathrm{b}}$ & $<0.01 \pm 0^{\mathrm{b}}$ & $<0.01 \pm 0^{\mathrm{b}}$ & $<0.02 \pm 0^{\mathrm{b}}$ & $0.7 \pm 0.1^{\mathrm{a}}$ & 0.7 \\
\hline C8:0 & $1.2 \pm 0.2^{\mathrm{b}}$ & ND & $8.4 \pm 0.3^{\mathrm{a}}$ & ND & ND & $0.2 \pm 0.5^{c}$ & ND \\
\hline $\mathrm{C} 10: 0$ & ND & ND & $7.0 \pm 0.8^{\mathrm{a}}$ & ND & ND & $0.1 \pm 0.01^{\mathrm{b}}$ & $\mathrm{ND}$ \\
\hline $\mathrm{C} 12: 0$ & $3.5 \pm 0.8^{\mathrm{b}}$ & ND & $46.8 \pm 1.1^{\mathrm{a}}$ & ND & $0.01 \pm 0^{c}$ & $0.1 \pm 0.02^{\mathrm{c}}$ & 0.1 \\
\hline $\mathrm{C} 14: 0$ & ND & $5.2 \pm 0.9^{b}$ & $22.8 \pm 1.1^{\mathrm{a}}$ & $5.9 \pm 0.8^{\mathrm{b}}$ & $0.1 \pm 0^{\mathrm{d}}$ & $1.4 \pm 0.1 \mathrm{c}$ & 3.3 \\
\hline C16:0 & $7.4 \pm 0.6^{\mathrm{d}}$ & $11.8 \pm 0.5^{\mathrm{b}}$ & $2.0 \pm 0.3^{\mathrm{e}}$ & $11.8 \pm 1.0^{\mathrm{b}}$ & $8.5 \pm 0.1^{\mathrm{c}}$ & $23.9 \pm 0.7^{\mathrm{a}}$ & 23.7 \\
\hline C18:0 & $5.3 \pm 0.4^{c}$ & $8.4 \pm 0.5^{b}$ & $2.3 \pm 0.2^{\mathrm{d}}$ & $7.9 \pm 1.0^{\mathrm{b}}$ & $2.0 \pm 0.02^{\mathrm{d}}$ & $12.8 \pm 1.3^{\mathrm{a}}$ & 13.1 \\
\hline $\mathrm{C} 20: 0$ & $1.1 \pm 0.1^{\mathrm{b}}$ & $18.4 \pm 0 .^{5 \mathrm{a}}$ & $0.2 \pm 0.1^{\mathrm{b}}$ & $18.7 \pm 1.6^{\mathrm{a}}$ & $0.4 \pm 0^{\mathrm{b}}$ & $0.1 \pm 0.1^{\mathrm{b}}$ & 0.1 \\
\hline C16:1 & ND & ND & ND & ND & $0.4 \pm 0^{\mathrm{b}}$ & $2.5 \pm 0.2^{\mathrm{a}}$ & 4.0 \\
\hline C18:1 & $31.9 \pm 2.2^{\mathrm{b}}$ & $53.8 \pm 1.2^{\mathrm{a}}$ & $7.0 \pm 0.3^{\mathrm{d}}$ & $53.6 \pm 1.5^{\mathrm{a}}$ & $10.0 \pm 0.4^{\mathrm{c}}$ & $33.4 \pm 2.9^{b}$ & 42.5 \\
\hline $\mathrm{C} 18: 1 \mathrm{n} 7$ & ND & ND & $0.03 \pm 0.07^{\mathrm{c}}$ & ND & $2.0 \pm 0.02^{\mathrm{b}}$ & $4.6 \pm 0.4^{\mathrm{a}}$ & 6.5 \\
\hline C20:1 & $1.1 \pm 0.1^{\mathrm{a}}$ & ND & $0.2 \pm 0.04^{c}$ & ND & $0.2 \pm 0^{c}$ & $0.6 \pm 0.04^{\mathrm{b}}$ & 0.4 \\
\hline C18:2 & $34.6 \pm 3.4^{\mathrm{a}}$ & $2.5 \pm 0.1^{\mathrm{c}}$ & $2.1 \pm 1.0^{\mathrm{c}}$ & $2.4 \pm 0.3^{\mathrm{c}}$ & $34.2 \pm 0.7^{\mathrm{a}}$ & $14.4 \pm 1.4^{\mathrm{b}}$ & 2.3 \\
\hline $\mathrm{C} 18: 3$ & $12.5 \pm 0.5^{\mathrm{b}}$ & ND & $0.7 \pm 0.1^{\mathrm{d}}$ & ND & $41.9 \pm 0.5^{\mathrm{a}}$ & $1.8 \pm 0.6^{\mathrm{c}}$ & 0.4 \\
\hline $\mathrm{C} 20: 4$ & $0.4 \pm 0.03^{\mathrm{b}}$ & ND & $0.1 \pm 0.1^{\mathrm{b}}$ & ND & $0.4 \pm 0^{\mathrm{b}}$ & $3.6 \pm 2.5^{\mathrm{a}}$ & 0.2 \\
\hline Total Saturated & $19.6 \pm 2.6$ & $43.7 \pm 5.0$ & $89.9 \pm 14.8$ & $44.1 \pm 5.1$ & $11.0 \pm 2.7$ & $38.9 \pm 8.0$ & 42.0 \\
\hline TotalMono-unsaturated & $33.0 \pm 15.3$ & $53.8 \pm 28.1$ & $7.2 \pm 3.4$ & $53.6 \pm 28.0$ & $10.6 \pm 4.7$ & $36.5 \pm 15.6$ & 55.2 \\
\hline TotalPoly-unsaturated & $47.5 \pm 11.7$ & $2.5 \pm 0.1$ & $3.0 \pm 0.8$ & $2.4 \pm 0.3$ & $78.4 \pm 16.6$ & $24.8 \pm 4.7$ & 2.9 \\
\hline
\end{tabular}

Table 8: Cholesterol content $(\mathrm{mg} / \mathrm{g} \pm \mathrm{SD})$ and fatty acid $(\%$ total $\mathrm{FA} \pm \mathrm{SD}$ ) profile of previous and current formulations of raw Beyond Meat Burger (BMB1 and BMB2), Impossible Foods Burger (IFB1 and IFB2), Black Bean Burger (BBB), 80/20 Ground Pork (GP), and 80/20 Ground Beef (GB).

Note: ${ }^{\mathrm{a}-\mathrm{d}}$ Means within a row with different subscripts differ statistically $(\mathrm{P}<0.05)$.

*Data collected from USDA nutrient database [19].

ND: Not Detected.

$<$ : Sample levels were not identified above the analysis thresholds for detection.

\begin{tabular}{|c|c|c|c|c|c|c|c|}
\hline Component & BMB1 & BMB2 & IFB1 & IFB2 & BBB & GP & GB* \\
\hline Cholesterol & $<0.01 \pm 0^{\mathrm{b}}$ & $<0.01 \pm 0^{\mathrm{b}}$ & $<0.01 \pm 0^{\mathrm{b}}$ & $<0.01 \pm 0^{\mathrm{b}}$ & $<0.02 \pm 0^{\mathrm{b}}$ & $0.9 \pm 0.1^{\mathrm{a}}$ & 0.9 \\
\hline C $8: 0$ & $1.1 \pm 0.1^{\mathrm{b}}$ & ND & $6.7 \pm 3.3^{\mathrm{a}}$ & ND & ND & ND & ND \\
\hline C10:0 & ND & ND & $5.8 \pm 2.8^{\mathrm{a}}$ & ND & ND & $0.1 \pm 0.01^{\mathrm{b}}$ & ND \\
\hline $\mathrm{C} 12: 0$ & $2.8 \pm 0.4^{\mathrm{b}}$ & ND & $39.0 \pm 19.1^{\mathrm{a}}$ & ND & $0.01 \pm 0^{\mathrm{b}}$ & $0.1 \pm 0.02^{\mathrm{b}}$ & 0.1 \\
\hline C14:0 & ND & $5.2 \pm 1.2^{\mathrm{b}}$ & $19.2 \pm 7.9^{\mathrm{a}}$ & $5.5 \pm 0.7^{b}$ & $0.1 \pm 0^{\mathrm{b}}$ & $1.3 \pm 0.1^{\mathrm{b}}$ & 3.2 \\
\hline C16:0 & $7.5 \pm 0.7^{c}$ & $12.0 \pm 1.1^{\mathrm{b}}$ & $1.8 \pm 0.9^{\mathrm{d}}$ & $11.9 \pm 0.7^{b}$ & $7.6 \pm 1.2^{\mathrm{c}}$ & $24.2 \pm 0.9^{\mathrm{a}}$ & 24.0 \\
\hline C18:0 & $5.4 \pm 0.3^{\mathrm{c}}$ & $8.2 \pm 0.9^{b}$ & $2.0 \pm 1.0^{\mathrm{d}}$ & $8.9 \pm 0.6^{b}$ & $2.0 \pm 0.1^{\mathrm{d}}$ & $13.0 \pm 1.3^{\mathrm{a}}$ & 13.4 \\
\hline C20:0 & $1.1 \pm 0.1^{\mathrm{b}}$ & $17.6 \pm 3.2^{\mathrm{a}}$ & $0.2 \pm 0.1^{\mathrm{b}}$ & $16.2 \pm 2.8^{\mathrm{ab}}$ & $0.4 \pm 0.01^{\mathrm{b}}$ & $0.1 \pm 0.1^{\mathrm{b}}$ & 0.1 \\
\hline C16:1 & ND & ND & ND & ND & $0.4 \pm 0.01^{\mathrm{b}}$ & $2.4 \pm 0.3^{\mathrm{a}}$ & 4.1 \\
\hline C18:1 & $32.2 \pm 1.8^{\mathrm{b}}$ & $54.5 \pm 1.4^{\mathrm{a}}$ & $6.2 \pm 3.1^{\mathrm{d}}$ & $55.0 \pm 2.4^{\mathrm{ab}}$ & $10.1 \pm 0.5^{\mathrm{c}}$ & $33.3 \pm 2.6^{\mathrm{b}}$ & 43.0 \\
\hline $\mathrm{C} 18: 1 \mathrm{n} 7$ & ND & ND & $0.0 \pm 0.1^{\mathrm{c}}$ & ND & $2.0 \pm 0.1^{\mathrm{b}}$ & $4.6 \pm 0.4^{\mathrm{a}}$ & 4.9 \\
\hline $\mathrm{C} 20: 1 \mathrm{n} 9$ & $1.1 \pm 0.1^{\mathrm{a}}$ & ND & $0.2 \pm 0.1^{\mathrm{c}}$ & ND & $0.2 \pm 0.01^{\mathrm{c}}$ & $0.6 \pm 0.03^{\mathrm{b}}$ & 0.4 \\
\hline C18:2 & $35.4 \pm 2.1^{\mathrm{a}}$ & $2.6 \pm 0.1^{\mathrm{c}}$ & $2.1 \pm 1.1^{\mathrm{c}}$ & $2.6 \pm 0.3^{c}$ & $35.2 \pm 0.9^{\mathrm{a}}$ & $14.1 \pm 1.7^{\mathrm{b}}$ & 2.5 \\
\hline C18:3 & $11.8 \pm 1.0^{\mathrm{b}}$ & ND & $0.7 \pm 0.4^{c}$ & ND & $41.5 \pm 0.8^{\mathrm{a}}$ & $1.8 \pm 0.6^{\mathrm{c}}$ & 0.4 \\
\hline $\mathrm{C} 20: 4$ & $0.4 \pm 0.03^{\mathrm{b}}$ & ND & $0.1 \pm 0.1^{\mathrm{b}}$ & ND & $0.4 \pm 0.01^{\mathrm{b}}$ & $3.7 \pm 2.5^{\mathrm{a}}$ & 0.3 \\
\hline Total Saturated & $19.1 \pm 2.6$ & $43.0 \pm 5.0$ & $74.6 \pm 14.0$ & $42.4 \pm 4.3$ & $10.1 \pm 2.4$ & $39.2 \pm 8.2$ & 42.5 \\
\hline Total Mono-unsaturated & $33.3 \pm 15.4$ & $54.5 \pm 28.5$ & $6.4 \pm 3.4$ & $55.0 \pm 28.8$ & $10.7 \pm 4.8$ & $36.2 \pm 15.5$ & 54.3 \\
\hline Total Poly-unsaturated & $47.7 \pm 11.9$ & $2.6 \pm 0.1$ & $3.0 \pm 0.8$ & $2.6 \pm 0.3$ & $79.1 \pm 16.7$ & $24.6 \pm 4.7$ & 3.2 \\
\hline
\end{tabular}

Table 9: Cholesterol content ( $\mathrm{mg} / \mathrm{g} \pm \mathrm{SD}$ ) and fatty acid (\% total FA \pm SD) profile of cooked Beyond Meat Burger (BMB1 and BMB2), Impossible Foods Burger (IFB1 and IFB2), Black Bean Burger (BBB), 80/20 Ground Pork (GP), and 80/20 Ground Beef (GB).

Note: ${ }^{\mathrm{a}-\mathrm{d}}$ Means within a row with different subscripts differ statistically $(\mathrm{P}<0.05)$.

*Data collected from USDA nutrient database [20].

ND: Not Detected.

$<$ : Sample levels were not identified above the analysis thresholds for detection. 
Citation: Swing CJ, Thompson TW, Guimaraes O, Geornaras I, Engle TE, et al. (2021) Nutritional Composition of Novel Plant-Based Meat Alternatives and Traditional Animal-Based Meats. J Food Sci Nutr 7: 109.

- Page 8 of 11 -

\begin{tabular}{|c|c|c|c|c|c|c|c|}
\hline Component & BMB1 & BMB2 & IFB1 & IFB2 & BBB & GP & GB* \\
\hline Arginine & $15.32 \pm 0.55^{\mathrm{a}}$ & $16.5 \pm 1.89^{\mathrm{a}}$ & $9.17 \pm 0.79^{c}$ & $11.4 \pm 0.66^{\mathrm{b}}$ & $8.16 \pm 0.3^{c}$ & $11.08 \pm 0.72^{\mathrm{b}}$ & 11.18 \\
\hline Cystine & $2.45 \pm 0.17^{\mathrm{c}}$ & $2.55 \pm 0.27^{\mathrm{c}}$ & $6.22 \pm 0.61^{\mathrm{a}}$ & $4.42 \pm 0.25^{\mathrm{b}}$ & $2.58 \pm 0 .{ }^{3 c}$ & $1.83 \pm 0.24^{\mathrm{d}}$ & 1.77 \\
\hline Glutamic Acid & $30.1 \pm 0.93^{c}$ & $33.17 \pm 3.57^{\mathrm{c}}$ & $69.57 \pm 3.39^{\mathrm{a}}$ & $37.87 \pm 2.2^{\mathrm{b}}$ & $29.87 \pm 1.66^{c}$ & $23.72 \pm 2.24^{\mathrm{d}}$ & 25.75 \\
\hline Glycine & $7.36 \pm 0.13^{\mathrm{cd}}$ & $8.06 \pm 0.92^{\mathrm{bc}}$ & $8.85 \pm 0.3^{b}$ & $7.09 \pm 0.37^{\mathrm{d}}$ & $4.7 \pm 0.18^{\mathrm{e}}$ & $9.75 \pm 0.66^{\mathrm{a}}$ & 11.66 \\
\hline Proline & $7.96 \pm 0.27^{\mathrm{c}}$ & $8.89 \pm 1.03^{\mathrm{bc}}$ & $21.97 \pm 1.01^{\mathrm{a}}$ & $8.34 \pm 0.51^{\mathrm{c}}$ & $9.8 \pm 0.38^{\mathrm{b}}$ & $7.93 \pm 0.53^{\mathrm{c}}$ & 8.75 \\
\hline Histidine & $4.24 \pm 0.08^{\mathrm{bc}}$ & $4.86 \pm 0.54^{\mathrm{ab}}$ & $4.08 \pm 0.17^{\mathrm{c}}$ & $3.85 \pm 0.2^{\mathrm{c}}$ & $3.06 \pm 0.06^{\mathrm{d}}$ & $5.46 \pm 0.79^{\mathrm{a}}$ & 5.58 \\
\hline Isoleucine & $8.74 \pm 0.14^{\mathrm{ab}}$ & $9.41 \pm 1.04^{\mathrm{a}}$ & $9.19 \pm 0.27^{\mathrm{a}}$ & $7.82 \pm 0.46^{\mathrm{bc}}$ & $6.08 \pm 0.25^{\mathrm{d}}$ & $7.28 \pm 0.78^{c}$ & 7.59 \\
\hline Leucine & $15.3 \pm 0.24^{\mathrm{a}}$ & $16.52 \pm 1.73^{\mathrm{a}}$ & $16.68 \pm 0.53^{\mathrm{a}}$ & $13 \pm 0.72^{\mathrm{b}}$ & $10.75 \pm 0.41^{c}$ & $12.7 \pm 1.2^{\mathrm{b}}$ & 13.39 \\
\hline Lysine & $13.28 \pm 0.36^{\mathrm{a}}$ & $12.82 \pm 1.58^{\mathrm{a}}$ & $7.63 \pm 0.33^{c}$ & $10.29 \pm 0.81^{\mathrm{b}}$ & $6.82 \pm 0.12^{\mathrm{c}}$ & $13.28 \pm 1^{\mathrm{a}}$ & 14.23 \\
\hline Methionine & $1.61 \pm 0.13^{\mathrm{d}}$ & $2.53 \pm 0.38^{\mathrm{c}}$ & $3.28 \pm 0.13^{\mathrm{b}}$ & $2.01 \pm 0.15^{\mathrm{d}}$ & $2.79 \pm 0.13^{\mathrm{c}}$ & $4.3 \pm 0.45^{\mathrm{a}}$ & 4.42 \\
\hline Phenylalanine & $9.87 \pm 0.18^{\mathrm{b}}$ & $11.04 \pm 1.09^{\mathrm{a}}$ & $11.82 \pm 0.35^{\mathrm{a}}$ & $8.65 \pm 0.51^{\mathrm{c}}$ & $7.15 \pm 0 . .^{2 \mathrm{~d}}$ & $6.3 \pm 0.56^{\mathrm{d}}$ & 6.7 \\
\hline Threonine & $6.67 \pm 0.13^{\mathrm{a}}$ & $7.3 \pm 0.86^{a}$ & $6.98 \pm 0.2^{\mathrm{a}}$ & $6.57 \pm 0.37^{\mathrm{a}}$ & $5.03 \pm 0.18^{\mathrm{b}}$ & $7.1 \pm 0.7^{\mathrm{a}}$ & 6.65 \\
\hline Tryptophan & $1.67 \pm 0.06^{\mathrm{c}}$ & $1.75 \pm 0.07^{\mathrm{bc}}$ & $2.29 \pm 0.1^{\mathrm{a}}$ & $2.26 \pm 0.08^{\mathrm{a}}$ & $1.7 \pm 0.08^{c}$ & $1.9 \pm 0.12^{\mathrm{b}}$ & 0.87 \\
\hline Valine & $9.12 \pm 0.19^{\mathrm{b}}$ & $10.4 \pm 1.11^{\mathrm{a}}$ & $10.52 \pm 0.21^{\mathrm{a}}$ & $8.17 \pm 0.48^{\mathrm{bc}}$ & $6.86 \pm 0.14^{\mathrm{d}}$ & $7.81 \pm 0.74^{\mathrm{cd}}$ & 8.44 \\
\hline Alanine & $7.93 \pm 0.19^{b}$ & $9.12 \pm 1.08^{\mathrm{a}}$ & $6.66 \pm 0.22^{\mathrm{cd}}$ & $7.7 \pm 0.47^{\mathrm{bc}}$ & $5.82 \pm 0.28^{\mathrm{d}}$ & $10.14 \pm 0.9^{\mathrm{a}}$ & 10.76 \\
\hline Aspartic Acid & $21.17 \pm 0.49^{\mathrm{a}}$ & $22.17 \pm 2.4^{\mathrm{a}}$ & $13.27 \pm 0.4^{c}$ & $18.63 \pm 1.05^{\mathrm{b}}$ & $13.4 \pm 0.5^{\mathrm{c}}$ & $14.68 \pm 1.41^{\mathrm{c}}$ & 15.47 \\
\hline Serine & $9.34 \pm 0.23^{\mathrm{bc}}$ & $10.16 \pm 1.18^{\mathrm{ab}}$ & $10.75 \pm 0.42^{\mathrm{a}}$ & $8.39 \pm 0.47^{\mathrm{c}}$ & $7.27 \pm 0.29^{\mathrm{d}}$ & $6.38 \pm 0.52^{\mathrm{d}}$ & 6.88 \\
\hline Total essential & $70.5 \pm 4.56$ & $76.63 \pm 4.74$ & $72.47 \pm 4.38$ & $62.62 \pm 3.54$ & $50.24 \pm 2.66$ & $66.13 \pm 3.56$ & 67.87 \\
\hline $\begin{array}{l}\text { Total conditionally-es- } \\
\text { sential** }\end{array}$ & $70.24 \pm 9.19$ & $77.42 \pm 10.21$ & $124.62 \pm 22.77$ & $75.61 \pm 11.69$ & $60.42 \pm 9.31$ & $59.82 \pm 7$ & 64.39 \\
\hline Total non-essential ${ }^{* * *}$ & $38.44 \pm 6.12$ & $41.45 \pm 6.29$ & $30.68 \pm 2.82$ & $34.72 \pm 5.19$ & $26.49 \pm 3.4$ & $31.2 \pm 3.62$ & 33.11 \\
\hline
\end{tabular}

Table 10: Amino Acid composition (mg/g $\pm \mathrm{SD}$ ) of two formulations of raw Beyond Meat Burger (BMB1 and BMB2), Impossible Foods Burger (IFB1 and IFB2), Black Bean Burger (BBB), 80/20 Ground Pork (GP), and 80/20 Ground Beef (GB).

Note: ${ }^{\text {ae }}$ Means within a row with different subscripts differ statistically $(\mathrm{P}<0.05)$.

*Data collected from USDA nutrient database [19].

**Includes the following: arginine, histidine, cystine, glycine, tyrosine, and proline.

***Glutamic acid, alanine, and aspartic acid

\begin{tabular}{|c|c|c|c|c|c|c|c|}
\hline Component & BMB1 & BMB2 & IFB1 & IFB2 & BBB & GP & $\mathbf{G B}^{*}$ \\
\hline Arginine & $16.8 \pm 1.1^{\mathrm{b}}$ & $20.2 \pm 0.9^{\mathrm{a}}$ & $9.7 \pm 0.6^{\mathrm{d}}$ & $13.8 \pm 0.6^{c}$ & $8.8 \pm 0.5^{\mathrm{d}}$ & $15.0 \pm 1.1^{\mathrm{c}}$ & 16.8 \\
\hline Cystine & $2.7 \pm 0.2^{\mathrm{c}}$ & $3.1 \pm 0.1^{\mathrm{c}}$ & $7.1 \pm 0.6^{\mathrm{a}}$ & $4.9 \pm 0.2^{\mathrm{b}}$ & $3 \pm 0.5^{c}$ & $2.5 \pm 0.2^{\mathrm{c}}$ & 2.7 \\
\hline GlutamicAcid & $33.6 \pm 1.7^{\mathrm{c}}$ & $40.8 \pm 1.5^{\mathrm{b}}$ & $80.9 \pm 5.0^{\mathrm{a}}$ & $45.2 \pm 1.2^{\mathrm{b}}$ & $34.7 \pm 5.2^{\mathrm{c}}$ & $32.1 \pm 3.0^{c}$ & 38.6 \\
\hline Glycine & $8.2 \pm 0.4^{\mathrm{c}}$ & $9.9 \pm 0.5^{\mathrm{bc}}$ & $10.2 \pm 0.5^{b}$ & $8.6 \pm 0.2^{\mathrm{bc}}$ & $5.3 \pm 0.4^{\mathrm{d}}$ & $13.0 \pm 2.2^{\mathrm{a}}$ & 17.5 \\
\hline Tyrosine & $7.7 \pm 0.4^{\mathrm{b}}$ & $10.1 \pm 0.5^{\mathrm{a}}$ & $10.0 \pm 0.5^{\mathrm{a}}$ & $7.8 \pm 0.2^{\mathrm{b}}$ & $6.0 \pm 0.5^{\mathrm{c}}$ & $7.4 \pm 0.5^{\mathrm{b}}$ & 7.9 \\
\hline Histidine & $4.8 \pm 0.2^{\mathrm{c}}$ & $5.9 \pm 0.3^{\mathrm{b}}$ & $4.8 \pm 0.3^{\mathrm{c}}$ & $4.7 \pm 0.2^{\mathrm{c}}$ & $3.5 \pm 0.3^{\mathrm{d}}$ & $7.6 \pm 0.6^{\mathrm{a}}$ & 8.4 \\
\hline Isoleucine & $9.8 \pm 0.5^{\mathrm{bc}}$ & $11.7 \pm 0.5^{\mathrm{a}}$ & $10.7 \pm 0.6^{\mathrm{b}}$ & $9.5 \pm 0.2^{\mathrm{c}}$ & $6.9 \pm 0.5^{\mathrm{d}}$ & $10.0 \pm 0.7^{\mathrm{bc}}$ & 11.4 \\
\hline Leucine & $17.2 \pm 0.8^{\mathrm{bc}}$ & $20.3 \pm 0.8^{\mathrm{a}}$ & $19.5 \pm 1.0^{\mathrm{a}}$ & $15.8 \pm 0.4^{c}$ & $12.4 \pm 0.9^{\mathrm{d}}$ & $17.5 \pm 1.3^{\mathrm{bc}}$ & 20.1 \\
\hline Lysine & $14.5 \pm 0.7^{\mathrm{b}}$ & $15.6 \pm 0.6^{\mathrm{b}}$ & $8.5 \pm 0.5^{\mathrm{d}}$ & $12.2 \pm 0.6^{\mathrm{c}}$ & $7.5 \pm 0.4^{\mathrm{d}}$ & $18.2 \pm 1.1^{\mathrm{a}}$ & 21.3 \\
\hline Methionine & $1.8 \pm 0.3^{\mathrm{e}}$ & $3 \pm 0.1^{\mathrm{c}}$ & $3.8 \pm 0.2^{\mathrm{b}}$ & $2.4 \pm 0.1^{\mathrm{d}}$ & $3.3 \pm 0.2^{\mathrm{c}}$ & $5.9 \pm 0 . .^{5 a}$ & 6.6 \\
\hline Threonine & $7.4 \pm 0.4^{\mathrm{b}}$ & $8.9 \pm 0.4^{\mathrm{a}}$ & $8.0 \pm 0.4^{\mathrm{b}}$ & $7.9 \pm 0.2^{\mathrm{b}}$ & $5.7 \pm 0.3^{\mathrm{c}}$ & $9.6 \pm 0.7^{\mathrm{a}}$ & 10.0 \\
\hline Tryptophan & $2.0 \pm 0.2^{\mathrm{c}}$ & $2.4 \pm 0.1^{\mathrm{b}}$ & $2.6 \pm 0.1^{\mathrm{ab}}$ & $2.6 \pm 0.1^{\mathrm{ab}}$ & $1.8 \pm 0.2^{\mathrm{c}}$ & $2.7 \pm 0.2^{\mathrm{a}}$ & 1.3 \\
\hline Valine & $10.0 \pm 0.5^{\mathrm{b}}$ & $12.8 \pm 0.5^{\mathrm{a}}$ & $11.9 \pm 0.5^{\mathrm{a}}$ & $9.9 \pm 0.2^{\mathrm{b}}$ & $7.7 \pm 0.6^{c}$ & $10.7 \pm 0.9^{b}$ & 12.6 \\
\hline Alanine & $9.0 \pm 0.4^{\mathrm{c}}$ & $11.1 \pm 0.5^{b}$ & $7.8 \pm 0.4^{\mathrm{d}}$ & $9.2 \pm 0.3^{\mathrm{c}}$ & $6.6 \pm 0.3^{d}$ & $14.0 \pm 1.4^{\mathrm{a}}$ & 16.1 \\
\hline Aspartic Acid & $23.9 \pm 1.3^{b}$ & $27.2 \pm 1.0^{\mathrm{a}}$ & $15.6 \pm 1.0^{\mathrm{d}}$ & $22.4 \pm 0.6^{\mathrm{b}}$ & $15.2 \pm 0.8^{\mathrm{d}}$ & $20.3 \pm 1.6^{c}$ & 23.2 \\
\hline Serine & $10.3 \pm 0.5^{b}$ & $12.3 \pm 0.6^{\mathrm{a}}$ & $12.4 \pm 0.7^{\mathrm{a}}$ & $10.0 \pm 0.4^{\mathrm{b}}$ & $8.3 \pm 0.6^{\mathrm{c}}$ & $8.6 \pm 0.7^{\mathrm{c}}$ & 10.3 \\
\hline Total essential & $78.2 \pm 5.1$ & $94.2 \pm 5.7$ & $83.2 \pm 5.1$ & $75.4 \pm 4.3$ & $56.8 \pm 3.1$ & $90.7 \pm 4.8$ & 101.7 \\
\hline
\end{tabular}


Citation: Swing CJ, Thompson TW, Guimaraes O, Geornaras I, Engle TE, et al. (2021) Nutritional Composition of Novel Plant-Based Meat Alternatives and Traditional Animal-Based Meats. J Food Sci Nutr 7: 109.

- Page 9 of $11 \bullet$

\begin{tabular}{|c|c|c|c|c|c|c|c|}
\hline Total conditionally-essential** & $78.0 \pm 10.3$ & $95.0 \pm 12.4$ & $143.2 \pm 26.7$ & $90.4 \pm 13.9$ & $69.3 \pm 11.1$ & $81.1 \pm 9.5$ & 96.5 \\
\hline Total non-essential*** & $43.1 \pm 7.0$ & $50.6 \pm 7.6$ & $35.7 \pm 3.4$ & $41.7 \pm 6.2$ & $30.1 \pm 3.9$ & $42.9 \pm 5.0$ & 49.6 \\
\hline
\end{tabular}

Table 11: Amino Acid composition (mg/g \pm SD) of two formulations of cooked Beyond Meat Burger (BMB1 and BMB2), Impossible Foods Burger (IFB1 and IFB2), Black Bean Burger (BBB), 80/20 Ground Pork (GP), and 80/20 Ground Beef (GB).

Note: ${ }^{\text {a-e }}$ Means within a row with different subscripts differ statistically $(\mathrm{P}<0.05)$

*Data collected from USDA nutrient database [20].

**Includes the following: arginine, histidine, cystine, glycine, tyrosine, and proline.

***Glutamic acid, alanine, and aspartic acid.

\section{Disclosure statement}

The authors declare that they have no conflicts of interest. Use of trade names in this publication does not imply endorsement or criticism by Colorado State University of those or similar products not mentioned.

Mention of a proprietary product does not constitute a guarantee or warranty of the products by Colorado State University or the authors and does not imply its approval to the exclusion of other products that may also be suitable.

\section{Data Availability}

All data generated or analyzed during this research are included in this manuscript.

\section{References}

1. Ritchie H, Roser M (2017) Micronutrient Deficiency. Our World in Data.

2. United Nations (2020) Progress towards the Sustainable Development Goals, Report of the Secretary-General. Economic and Social Council.

3. United Nations Children's Fund (2020) Malnutrition in Children. United

4. World Health Organization (2020) Obesity and overweight. World Health Organization.

5. Henchion M, McCarthy M, Resconi VC, Troy D (2014) Meat consumption: Trends and quality matters. Meat Sci 98: 561-568.

6. Steinfeld H, Gerber P, Wassenaar T, Castel V, Rosales M, et al. (2006) Livestock's long shadow: Environmental issues and options. Food and Agriculture Organization of the United Nations.

7. Bruinsma J (2009) The resource outlook to 2050: By how much do land, water and crop yields need to increase by 2050 . Expert meeting on how to feed the world 2050: 24-26.

8. Segovia-Siapco G, Sabaté J (2019) Health and sustainability outcomes of vegetarian dietary patterns: A revisit of the EPIC-Oxford and the Adventist Health Study-2 cohorts. Eur J Clin Nutr 72: 60-70.

9. Estell M, Hughes J, Grafenauer S (2021) Plant protein and plant-based meat alternatives: Consumer and nutrition professional attitudes and perceptions. Sustainability 13: 1478.

10. Shurtleff W, Aoyagi A (2014) History of Meat Alternatives (965 CE to 2014): Extensively Annotated Bibliography and Sourcebook. Soyinfo Center.

11. Bhandalkar S (2020) Meat Substitute Market Expected to Reach \$8.1 Billion by 2027. AMR.

12. Sha L, Xiong YL (2020) Plant protein-based alternatives of reconstructed meat: Science, technology, and challenges. Trends Food Sci Tech 102: 5161. Nations Children's Fund.

14. Gemede HF, Ratta N (2014) Antinutritional factors in plant foods: Potential health benefits and adverse effects. International J Nutr Food Sci 3: 284-289.

15. Hu FB, Otis BO, McCarthy G (2019) Can plant-based meat alternatives be part of a healthy and sustainable diet? JAMA 322: 1547-1548.

16. Deb-Choudhury S, Haines S, Harland D, Clerens S, van Koten C, et al. (2014) Effect of cooking on meat proteins: Mapping hydrothermal protein modification as a potential indicator of bioavailability. J Agric Food Chem 62: $8187-8196$

17. Czerwonka M, Tokarz A (2017) Iron in red meat-friend or foe. Meat Sci 123: $157-165$.

18. McAfee AJ, McSorley EM, Cuskelly GJ, Moss BW, Wallace JMW, et al. (2010) Red meat consumption: An overview of the risks and benefits. Meat Sci 84: 1-13.

19. United States Department of Agriculture (2020) Beef, Ground, $80 \%$ lean meat/ 20\% fat, raw (FDC ID: 174036; NDB Number: 23572).

20. United States Department of Agriculture (2020) Beef, ground, 80\% lean meat/ 20\% fat, patty, cooked, broiled (FDC ID: 17197; NDB Number: 23573).

21. United Sates Department of Health and Human Services, National Institute of Health (2020) Nutrient Recommendations: Dietary Reference Intakes (DRI).

22. Association of Official Analytical Chemist International (1995) Official methods of analysis of AOAC International, 16th edition. Volume 1.

23. Folch J, Lees M, Stanley GS (1957) A simple method for the isolation and purification of total lipides from animal tissues. J Biol Chem 226: 497-509.

24. Association of Official Analytical Chemist International (2006) Official methods of analysis 18th Edition, Association of Official Analytical Chemists.

25. Association of Official Analytical Chemist International (2011) Official methods of analysis 19th Edition. Arlington.

26. Njeru CA, McDowell LR, Shireman RM, Wilkinson NS, Rojas LX, et al. (1995) Assessment of vitamin E nutritional status in yearling beef heifers. Anim Sci J 73: 1440-1448.

27. Alosilla CE, McDowell LR, Wilkinson NS, Staples CR, Thatcher WW, et al. (2007) Bioavailability of vitamin A sources for cattle. Anim Sci J 85: 1235-1238.

28. Speek AJ, Schijver J, Schreurs WHP (1985) Vitamin E composition of some seed oils as determined by high- performance liquid chromatography with fluorometric detection. J Food Sci 50: 121-124.

29. Cort WM, Vincente TS, Waysek EH, Williams BD (1983) Vitamin E content of feedstuffs determined by high- performance liquid chromatographic fluorescence. J Agric Food Chem 31: 1130-1333.

30. McMurray CH, Blanchflower WJ, Rice DA (1980) Influence of extraction techniques on determination of $\alpha$-Tocopherol in animal feedstuffs. JAOAC 63: $1258-1261$ purification. Can J Biochem Physiol 37: 911-917. 
Citation: Swing CJ, Thompson TW, Guimaraes O, Geornaras I, Engle TE, et al. (2021) Nutritional Composition of Novel Plant-Based Meat Alternatives and Traditional Animal-Based Meats. J Food Sci Nutr 7: 109.

32. Parks P, Goins RE (1994) In situ preparation of fatty acid methyl esters for analysis of fatty acid composition in food. J Food Sci 59: 1262-1266.

33. Barkholt V, Jensen AL (1989) Amino acid analysis: determination of cysteine plus half-cystine in proteins after hydrochloric acid hydrolysis with a disulfide compound as additive. Anal Biochem 177: 318-322.

34. Henderson JW, Ricker RD, Bidlingmeyer BA, Woodward C (2000) Rapid accurate, sensitive, and reproducible HPLC analysis of amino acids, amino acid analysis using zorbax eclipse-AAA columns and the Agilent 1100 HPLC. Agilent Publication.

35. Henderson JW, Brooks A (2010) Improved Amino Acid Methods using Agilent ZORBAX Eclipse Plus C18 Columns for a Variety of Agilent LC Instrumentation and Separation Goals. Agilent Application Note.

36. Schuster R (1988) Determination of amino acids in biological, pharmaceutical, plant and food samples by automated precolumn derivatization and HPLC. J Chromatogr A 431: 271-284.

37. R Core Team (2020) R: A Language and Environment for Statistical Computing. Vienna, Austria.

38. John F, Sanford W (2019) An $\{\mathrm{R}\}$ Companion to Applied Regression Thousand Oaks CA.

39. Russell VL (2020) Emmeans: Estimated Marginal Means, aka LeastSquares Means.

40. National Academies of Sciences E, Oria M, Harrison M, Stallings VA (2019) Dietary Reference Intakes (DRIs): Tolerable Upper Intake Levels, Elements, Food and Nutrition Board, National Academies.

41. Desmond E (2006) Reducing salt: A challenge for the meat industry. Mea Sci 74: 188-196.

42. Hurrell RF, Juillerat MA, Reddy MB, Lynch SR, Dassenko SA, et al (1992) Soy protein, phytate, and iron absorption in humans. Am J Clin Nutr 56: 573-578.

43. Bohn L, Meyer AS, Rasmussen Søren K (2008) Phytate: Impact on environment and human nutrition. A challenge for molecular breeding. J Zhejiang Univ Sci B 9: 165-191

44. Reinhold JG, Faradji B, Abadi P, Ismail-Beigi F (1976) Decreased absorption of calcium, magnesium, zinc and phosphorus by humans due to increased fiber and phosphorus consumption as wheat bread. J Nutr 106: 493-503.

45. Gharibzahedi SMT, Jafari SM (2017) The importance of minerals in human nutrition: Bioavailability, food fortification, processing effects and nanoencapsulation. Trends in Food Sci Tech 62: 119-132.

46. Jeanes YM, Hall WL, Ellard S, Lee E, Lodge JK (2004) The absorption of vitamin $\mathrm{E}$ is influenced by the amount of fat in a meal and the food matrix. Br J Nutr 92: 575-579.

47. Slatopolsky E, Weerts C, Stokes T, Windus D, Delmez J (1986) Alternative phosphate binders in dialysis patients: Calcium carbonate. Semin Nephrol 6: $35-41$.

48. Lynch SR (2000) The effect of calcium on iron absorption. Nutr Res Rev 13: $141-158$.

49. Kies C (1987) Manganese bioavailability overview. In: Nutritional Bioavailability of Manganese. American Chemical Society, USA.

50. Troesch B, Egli I, Zeder C, Hurrell RF, de Pee S, et al. (2009) Optimization of a phytase-containing micronutrient powder with low amounts of highly bioavailable iron for in-home fortification of complementary foods. Am J Clin Nutr 89: 539-544.

51. Matkovic V, Ilich JZ, Andon MB, Hsieh LC, Tzagournis MA, et al. (1995) Urinary calcium, sodium, and bone mass of young females. Am J Clin Nutr 62: 417-425.

52. Juárez M, Dugan MER, Aldai N, Basarab JA, Baron VS, et al. (2012) Beef
53. Radimer K, Bindewald B, Hughes J, Ervin B, Swanson C, et al. (2004) Dietary supplement use by us adults: Data from the national health and nutrition examination survey, 1999-2000. Am J Epidemiol 160: 339-349.

54. Borel P, Preveraud D, Desmarchelier C (2013) Bioavailability of vitamin E in humans: An update. Nutr Rev 71: 319-331.

55. Farrer KTH (1957) Advances in food research, Academic Press. Massachusetts, USA.

56. Woodcock EA, Warthesen JJ, Labuza TP (1982) Riboflavin photochemical degradation in pasta measured by high performance liquid chromatography. J Food Sci 47: 545-549

57. Furuya EM, Warthesen JJ, Labuza TP (1984) Effects of water activity, light intensity, and physical structure of food on the kinetics of riboflavin photodegradation. J Food Sci 49: 526-528.

58. Pegg RB, Landen WO, Eitenmiller RR (2012) Vitamin Analysis. In: Food Analysis. Springer, Boston, USA

59. Ball GFM (2005) Vitamins in Foods: Analysis, Bioavailability, and Stability. CRC Press. Florida, USA.

60. Wall JS, Carpenter KJ (1988) Variation in availability of niacin in grain products.

61. Lii JFG, Ink SL, Sartain DB (1986) Degradation and binding to food proteins of vitamin B-6 compounds during thermal processing. J Food Sci 51 : 1345-1351.

62. Gadient M (1986) Effect of pelleting on nutritional quality of feed. Proceedings In Proceedings-Maryland Nutrition Conference for Feed Manufacturers (USA).

63. Reynolds RD (1988) Bioavailability of vitamin B-6 from plant foods. Am J Clin Nutr 48: 863-867.

64. Rindi G, Laforenza U (2000) Thiamine intestinal transport and related issues: Recent Aspects. Proc Soc Exp Biol Med 224: 246-255.

65. Sriram K, Manzanares W, Joseph K (2012) Thiamine in nutrition therapy Nutr Clin Pract 27: 41-50.

66. McDowell LR (2008) Vitamins in Animal and Human Nutrition. John Wiley \& Sons. New Jersey, USA.

67. Bhatnagar AS, Kumar PKP, Hemavathy J, Krishna AGG (2009) Fatty acid composition, oxidative stability, and radical scavenging activity of vegetable oil blends with coconut oil. JAOCS 86: 991-999.

68. Lipp M, Simoneau C, Ulberth F, Anklam E, Crews C, et al. (2001) Composition of genuine cocoa butter and cocoa butter equivalents. J Food Compos Anal 14: 399-408.

69. De Carvalho CCCR, Caramujo MJ (2018) The various roles of fatty acids Molecules 23: 2583

70. Roediger WEW (1994) Famine, fiber, fatty acids, and failed colonic absorption: Does fiber fermentation ameliorate diarrhea? JPEN 18: 4-8.

71. Fuse K, Bamba T, Hosoda S (1989) Effects of pectin on fatty acid and glucose absorption and on thickness of unstirred water layer in rat and human intestine. Digest Dis Sci 34: 1109-1116.

72. Gorissen SHM, Witard OC (2018) Characterizing the muscle anabolic potential of dairy, meat and plant-based protein sources in older adults. Proc Nutr Soc 77: 20-31.

73. Pownall TL, Udenigwe CC, Aluko RE (2010) Amino Acid Composition and Antioxidant Properties of Pea Seed (Pisum sativum L.) Enzymatic Protein Hydrolysate Fractions. J Agric Food Chem 58: 4712-4718.

74. Du M, Xie J, Gong B, Xu X, Tang W, et al. (2018) Extraction, physicochemical characteristics and functional properties of Mung bean protein Food Hydrocoll 76: 131-140.

75. Shih FF, Daigle KW (2000) Preparation and characterization of rice protein isolates. JAOCS 77: 885-889.

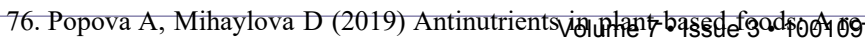
view. Open Biotechnol J 13. 
Citation: Swing CJ, Thompson TW, Guimaraes O, Geornaras I, Engle TE, et al. (2021) Nutritional Composition of Novel Plant-Based Meat Alternatives and Traditional Animal-Based Meats. J Food Sci Nutr 7: 109.

77. Mubarak AE (2005) Nutritional composition and antinutritional factors of mung bean seeds (Phaseolus aureus) as affected by some home traditional processes. Food Chem 89: 489-495.

78. Joint WHO/FAO/UNU Expert Consultation (2007) Protein and amino acid requirements in human nutrition. World Health Organization.

79. Burd NA, Beals JW, Martinez IG, Salvador AF, Skinner SK (2019) Foodfirst approach to enhance the regulation of post-exercise skeletal muscle protein synthesis and remodeling. Sports Med 49: 59-68.
80. Sarwar G (1997) The protein digestibility-corrected amino acid score method overestimates quality of proteins containing antinutritional factors and of poorly digestible proteins supplemented with limiting amino acids in rats. J Nutr 127: 758-764.

81. Mariotti F, Mahé S, Benamouzig R, Luengo C, Daré S, et al. (1999) Nutritional value of $[15 \mathrm{~N}]$-soy protein isolate assessed from ileal digestibility and postprandial protein utilization in humans. J Nutr 129: 992-1997. 


\section{$\mathbb{d}$ \\ Hнram}

Advances In Industrial Biotechnology | ISSN: 2639-5665

Advances In Microbiology Research | ISSN: 2689-694X

Archives Of Surgery And Surgical Education | ISSN: 2689-3126

Archives Of Urology

Archives Of Zoological Studies | ISSN: 2640-7779

Current Trends Medical And Biological Engineering

International Journal Of Case Reports And Therapeutic Studies | ISSN: 2689-310X

Journal Of Addiction \& Addictive Disorders | ISSN: 2578-7276

Journal Of Agronomy \& Agricultural Science | ISSN: 2689-8292

Journal Of AIDS Clinical Research \& STDs | ISSN: 2572-7370

Journal Of Alcoholism Drug Abuse \& Substance Dependence | ISSN: 2572-9594

Journal Of Allergy Disorders \& Therapy | ISSN: 2470-749X

Journal Of Alternative Complementary \& Integrative Medicine | ISSN: 2470-7562

Journal Of Alzheimers \& Neurodegenerative Diseases | ISSN: 2572-9608

Journal Of Anesthesia \& Clinical Care | ISSN: 2378-8879

Journal Of Angiology \& Vascular Surgery | ISSN: 2572-7397

Journal Of Animal Research \& Veterinary Science | ISSN: 2639-3751

Journal Of Aquaculture \& Fisheries | ISSN: 2576-5523

Journal Of Atmospheric \& Earth Sciences | ISSN: 2689-8780

Journal Of Biotech Research \& Biochemistry

Journal Of Brain \& Neuroscience Research

Journal Of Cancer Biology \& Treatment | ISSN: 2470-7546

Journal Of Cardiology Study \& Research | ISSN: 2640-768X

Journal Of Cell Biology \& Cell Metabolism | ISSN: 2381-1943

Journal Of Clinical Dermatology \& Therapy | ISSN: 2378-8771

Journal Of Clinical Immunology \& Immunotherapy | ISSN: 2378-8844

Journal Of Clinical Studies \& Medical Case Reports | ISSN: 2378-8801

Journal Of Community Medicine \& Public Health Care | ISSN: 2381-1978

Journal Of Cytology \& Tissue Biology | ISSN: 2378-9107

Journal Of Dairy Research \& Technology | ISSN: 2688-9315

Journal Of Dentistry Oral Health \& Cosmesis | ISSN: 2473-6783

Journal Of Diabetes \& Metabolic Disorders | ISSN: 2381-201X

Journal Of Emergency Medicine Trauma \& Surgical Care | ISSN: 2378-8798

Journal Of Environmental Science Current Research | ISSN: 2643-5020

Journal Of Food Science \& Nutrition | ISSN: 2470-1076

Journal Of Forensic Legal \& Investigative Sciences | ISSN: 2473-733X

Journal Of Gastroenterology \& Hepatology Research | ISSN: 2574-2566
Journal Of Genetics \& Genomic Sciences | ISSN: 2574-2485

Journal Of Gerontology \& Geriatric Medicine | ISSN: 2381-8662

Journal Of Hematology Blood Transfusion \& Disorders | ISSN: 2572-2999

Journal Of Hospice \& Palliative Medical Care

Journal Of Human Endocrinology | ISSN: 2572-9640

Journal Of Infectious \& Non Infectious Diseases | ISSN: 2381-8654

Journal Of Internal Medicine \& Primary Healthcare | ISSN: 2574-2493

Journal Of Light \& Laser Current Trends

Journal Of Medicine Study \& Research | ISSN: 2639-5657

Journal Of Modern Chemical Sciences

Journal Of Nanotechnology Nanomedicine \& Nanobiotechnology | ISSN: 2381-2044 Journal Of Neonatology \& Clinical Pediatrics | ISSN: 2378-878X

Journal Of Nephrology \& Renal Therapy | ISSN: 2473-7313

Journal Of Non Invasive Vascular Investigation | ISSN: 2572-7400

Journal Of Nuclear Medicine Radiology \& Radiation Therapy | ISSN: 2572-7419

Journal Of Obesity \& Weight Loss | ISSN: 2473-7372

Journal Of Ophthalmology \& Clinical Research | ISSN: 2378-8887

Journal Of Orthopedic Research \& Physiotherapy | ISSN: 2381-2052

Journal Of Otolaryngology Head \& Neck Surgery | ISSN: 2573-010X

Journal Of Pathology Clinical \& Medical Research

Journal Of Pharmacology Pharmaceutics \& Pharmacovigilance | ISSN: 2639-5649

Journal Of Physical Medicine Rehabilitation \& Disabilities | ISSN: 2381-8670

Journal Of Plant Science Current Research | ISSN: 2639-3743

Journal Of Practical \& Professional Nursing | ISSN: 2639-5681

Journal Of Protein Research \& Bioinformatics

Journal Of Psychiatry Depression \& Anxiety | ISSN: 2573-0150

Journal Of Pulmonary Medicine \& Respiratory Research | ISSN: 2573-0177

Journal Of Reproductive Medicine Gynaecology \& Obstetrics | ISSN: 2574-2574

Journal Of Stem Cells Research Development \& Therapy | ISSN: 2381-2060

Journal Of Surgery Current Trends \& Innovations | ISSN: 2578-7284

Journal Of Toxicology Current Research | ISSN: 2639-3735

Journal Of Translational Science And Research

Journal Of Vaccines Research \& Vaccination | ISSN: 2573-0193

Journal Of Virology \& Antivirals

Sports Medicine And Injury Care Journal | ISSN: 2689-8829

Trends In Anatomy \& Physiology | ISSN: 2640-7752

Submit Your Manuscript: https://www.heraldopenaccess.us/submit-manuscript 